\title{
Augmenting Neutralization breadth against Diverse HIV-1 by increasing the Ab-Ag interface on V2
}

Nan Gao ${ }^{1}$, Yanxin Gai ${ }^{1}$, Lina Meng ${ }^{1}$, Chu Wang ${ }^{1}$, Wei Wang ${ }^{2,3}$, Xiaojun $\mathrm{Li}^{4}$, Tiejun Gu${ }^{1}$, Mark K. Louder ${ }^{5}$, Nicole A. Doria-Rose ${ }^{5}$, Kevin Wiehe ${ }^{6}$, Alexandra F. Nazzari ${ }^{5}$, Adam S. Olia ${ }^{5}$, Jason Gorman ${ }^{5}$, Reda Rawi ${ }^{5}$, Wenmin $\mathrm{Wu}^{7}$, Clayton Smith ${ }^{7}$, Htet Khant ${ }^{7}$, Natalia de Val ${ }^{7}$, Bin $\mathrm{Yu}^{1}$, Junhong Luo ${ }^{8}$, Haitao Niu${ }^{8}$, Yaroslav Tsybovsky ${ }^{7}$, Huaxin Liao ${ }^{8}$, Thomas B. Kepler ${ }^{9}$, Peter D. Kwong ${ }^{5}$, John R. Mascola ${ }^{5}$, Chuan Qin ${ }^{2,3^{*}}$, Tongqing Zhou ${ }^{5^{*}}$, Xianghui Yu ${ }^{1,10^{*}}$, Feng Gao ${ }^{1,4,8^{*} \#}$

${ }^{1}$ National Engineering Laboratory for AIDS Vaccine, School of Life Sciences, Jilin University, Changchun, Jilin Province 130012, China.

${ }^{2}$ Institute of Laboratory Animal Science, Chinese Academy of Medical Sciences, Beijing 100021, China.

${ }^{3}$ Comparative Medicine Center, Peking Union Medical College, Beijing 100021, China.

${ }^{4}$ Department of Medicine, Duke University School of Medicine, Durham, North Carolina 27710, USA.

${ }^{5}$ Vaccine Research Center, National Institute of Allergy and Infectious Diseases, National Institutes of Health, Bethesda, MD 20892, USA

${ }^{6}$ Duke University Human Vaccine Institute, Duke University School of Medicine, Durham NC 27710, USA

${ }^{7}$ Cancer Research Technology Program Frederick National Laboratory for Cancer Research, Leidos Biomedical Research Inc., Frederick, MD 21701, 
USA

${ }^{8}$ School of Medicine, Jinan University, Guangzhou, Guangdong Province 510632, China.

${ }^{9}$ Department of Microbiology, Boston University, Boston, Massachusetts 02215 , USA.

${ }^{10}$ Key Laboratory for Molecular Enzymology and Engineering, the Ministry of Education, School of Life Sciences, Jilin University, Changchun, Jilin Province 130012, China.

Corresponding authors: qinchuan@pumc.edu.cn; tzhou@nih.gov;

xianghui@jlu.edu.cn; fgao@duke.edu

Lead contact: fgao@duke.edu 


\section{SUMMARY}

Understanding maturation pathways of broadly neutralizing antibodies (bnAbs) against HIV-1 in non-human primates can be highly informative for HIV1 vaccine development. We now obtained a lineage of J038 from Chinese rhesus macaques after 7-years of SHIV infection. J038 has short complementary determining loops and neutralizes $54 \%$ of global circulating HIV-1 strains. Its binding induces a unique "up" conformation for one of the V2 loops in the trimeric envelope glycoprotein (Env) and is heavily dependent on glycan, which provides nearly half of the binding surface. The unmutated common ancestor of the J038 lineage antibodies binds monomeric gp120 and neutralizes the autologous virus. Continuous maturation enhances neutralization potency and breadth of J038 lineage antibodies via expanding antibody-Env contact areas surrounding the core region contacted by germlineencoded residues. Developmental details and recognition features of J038 lineage antibodies revealed here provide a new pathway for maturation elicitation of V2-targeting bnAbs.

Keywords: HIV-1, SHIV, broadly neutralizing antibodies, maturation, nonhuman primate 


\section{Highlights}

- Long-term infected NHPs develop antibodies neutralizing up to $54 \%$ of HIV-1 strains

- Antibody J038 binds one V2 loop on HIV-1 Env trimer in a unique "up" position

- UCA of the J038 lineage effectively neutralizes the autologous virus

- J038 lineage antibodies mature through gradually increased contact to glycans 


\section{Introduction}

HIVIAIDS has affected 76 million people in the world since the start of the epidemic (UNAIDS, 2021). However, after over 30 years of search, an AIDS vaccine is still illusive. The RV144 phase III trial shows a $31 \%$ efficacy (RerksNgarm et al., 2009), but the recent failure of a similar phase III trial in Africa (HVTN 702) shows the extreme challenges in development of an effective AIDS vaccine (Gray et al., 2021). One major challenge is the extraordinarily high level of genetic variation among global circulating HIV-1 strains; up to $30 \%$ differences in the envelope glycoprotein (Env) which is the sole target for neutralizing antibodies (nAbs) (Malim and Emerman, 2001).. Thus, broadly neutralizing antibodies (bnAbs) against diverse HIV-1 strains should be required for a vaccine to effectively prevent HIV-1 infection by the global strains (Burton and Mascola, 2015; Haynes et al., 2019; Kwong and Mascola, 2018). Broad neutralization activity can be detected in the sera of $\sim 20 \% \mathrm{HIV}-1$ infected individuals after 2-4 years of infection (Hraber et al., 2014; Landais et al., 2016). Hundreds of bnAbs have been isolated from such individuals and structural analysis demonstrates that six main conserved sites (V2 apex, the CD4 binding site [CD4bs], V3-glycan, the membrane proximal external region [MPER], silence face and the gp120-gp41 interface, including the fusion peptide) on Env are targeted by bnAbs (Kwong and Mascola, 2018; Sok and Burton, 2018). Importantly, infusion of bnAbs in transgenic mice and non-human primates (NHPs) can prevent acquisition of SHIV infection (Gautam et al., 2016; Hessell and Haigwood, 2015; Pegu et al., 2017), indicating that bnAbs can effectively prevent HIV-1 infection if they are elicited by vaccines. The results from the Antibody-Mediated Prevention (AMP) trail shows that VRC01, a CD4bs bnAb, 
is $75.4 \%$ effective to prevent infection of VRC01 sensitive viruses, although overall it does not prevent acquisition of other HIV-1 isolates (Corey et al., 2021). A recent study demonstrates that high titer bnAbs, not $\mathrm{T}$ cell immunity or ADCC, is associated with protection of inquisition of SHIV infection (Pauthner et al., 2019). However, such potent and broad nAbs have not be successfully elicited by vaccines in NHPs or humans (Andrabi et al., 2018; Haynes et al., 2019).

Potent neutralization against autologous tier 2 virus has been elicited in animal models by current vaccine strategies (Havenar-Daughton et al., 2016; Sanders et al., 2015; Williams et al., 2017). Recent studies show that some levels of broad neutralization breadth can be achieved. The fusion peptide (FP) vaccine has elicited weak but cross-clade neutralizing activities in mice, guinea pigs and non-human primates (NHPs) (Kong et al., 2019; Xu et al., 2018). A vaccine approach using the $\mathrm{N}$-glycan removal trimers prime and boosting with gradual N-glycan restoration heterologous trimers on liposomes to elite broadly neutralizing responses in rabbits, in which an interface targeted monoclonal bnAb with $87 \%$ neutralization breadth was isolated (Dubrovskaya et al., 2019). Other studies show that V3-glycan targeted neutralizing antibodies with limited breadth can be elicited in rhesus macaques (Alam et al., 2017; Han et al., 2019; Sanders et al., 2015). These results show that neutralizing antibodies are likely be induced by vaccine strategies.

NHPs are the only model which can evaluate protection of inquisition of HIV-1 infection (Rahman and Robert-Guroff, 2019). However, little is known about if bnAbs with similar breadth and potency as those in humans can 
develop in NHPs and whether they developed in a similar manor as in humans (Roark et al., 2021; Wang et al., 2020). A better understanding of these questions will be critical for use of NHPs to evaluate the protection efficacy of HIV-1 Env-based vaccines. In our previous study, we have detected broad neutralization activities against HIV-1 in Chinese rhesus macaques after 6years of SHIV infection (Gao et al., 2018). Epitope mapping showed that the broad neutralization activity has multiple specificities, targeting V2, V3 and CD4bs that are commonly detected for bnAbs in humans (Gao et al., 2020; Gao et al., 2018). Here we isolated the multiple mAbs from one Chinese rhesus macaque using V2 differential baits. Biological and structural analyses of these mAbs showed that they are broadly neutralizing, uniquely bind to the V2 apex, and have a novel maturation pathway for broad neutralization. These findings will have important implications in understanding the differences in bnAb maturation in humans and NHPs and how to best utilize the NHP model to evaluate HIV-1 Env-based vaccines.

\section{Results}

\section{J038 lineage Abs broadly neutralize diverse HIV-1 strains}

We previously found that the plasmas from one SHIV ${ }_{1157 i p d 3 N 4-i n f e c t e d}$ Chinses rhesus macaque (G1015R) neutralized $65 \%$ of a panel of 17 tier 2 viruses by week 321 post infection (Gao et al., 2018). The G1015R plasma from week 350 still maintained broad neutralization activity, neutralizing $71 \%$ of the same panel of the 17 viruses (Figure 1A). Our previous epitope mapping results showed that many mutations (N160A, T162A and K169E) in the variable loop 
2 (V2) in Env rendered the virus resistant to the neutralization by the week 321 plasma, indicating that the V2-specific bnAbs might have been elicited in G1015R (Gao et al., 2020; Gao et al., 2018). To delineate if such bnAbs were elicited in G1015R, we used the peripheral blood mononuclear cells (PBMCs) collected at week 350 to sort 172 single memory B cells that specifically bound to V2 (positive for wild type A244 gp120 but negative for the mutant A244 carrying both N156K and N160K mutations) into individual wells in 96-well plates (Figure 1B).

The paired variable Ig heavy and light chains (VH and VL) were amplified from 44 single cells (Figure S1A). The recombinant protein for each Ab was expressed by co-transfecting the $\lg \mathrm{VH}$ and $\mathrm{VL}$ genes into the 293T cells. Twelve mAbs showed specific binding to autologous SHIV 1157ipd3N4 gp120 protein (Figure S1A). Sequence analysis showed that the $V(D) J$ sequences of six Abs (J029, J031, J033, J038, J040 and J044) were from the same gene family (Table S1). Maximum likelihood trees showed that the VH and VL genes from these six Abs evolved similarly, forming two distinct clades; clade A with four sequences and clade B with two sequences (Figure S1B). They all had 18 and 9 amino acids (AAs) in the complementarity-determining region 3 (CDR3) of $\mathrm{VH}$ and $\mathrm{VL}$ chains, respectively (Table S1). The average of somatic hypermutation (SHM) frequencies were $19.83 \%$ and $17 \%$ in $\mathrm{VH}$ and $\mathrm{VL}$, respectively. All but J044 bound to autologous 1157ipd3N4 gp120 and heterologous BG505 Env trimer at high affinity (Figure S2). The V(D)J sequences in the $\mathrm{VH}$ and $\mathrm{VL}$ genes had distinct origins in the other six Abs, indicating that they derived from independent germlines (Table S1). 
When tested with the same panel of 17 tier 2 viruses, all six members from the same lineage neutralized the viruses at various levels $(0.14$ to $48.29 \mu \mathrm{g} / \mathrm{ml})$, with the neutralization breadth ranging from $23.5 \%$ to $76.5 \%$ (Figure $1 \mathrm{C}$ ). Among them, two (J033 and J038) of three most evolved antibodies (Figure S1B) showed broadest neutralization activities (70.6\% and $76.5 \%$, respectively). The similar neutralization patterns between both mAbs and week 350 plasma suggested that both Abs represented the overall plasma neutralization activity well. In contrast, all other six mAbs did not neutralize any of those 17 viruses, except one case (J032 weakly neutralized 25710). All 12 mAbs potently neutralized autologous tier 2 virus 1157ipd3N4 (Siddappa et al., 2010) and they also neutralized tier 1 virus MW96526, except J013. None of them neutralized tier 1 virus 92RW020.2 and only three of them neutralized tier 1 virus SF162.SL. These results demonstrated that bnAbs representing the overall plasma neutralization activity were induced in G1015R after seven years of infection.

The neutralization potency and breadth of J038 were further assessed with a panel of 208 viruses representing global major circulating HIV-1 strains. Neutralization results from this large panel showed that J038 could broadly neutralize 112 viruses, or $54 \%$, at a maximum of concentration threshold of 50 $\mu \mathrm{g} / \mathrm{ml}$ (Figure 1D, Table S2). The median $\mathrm{IC}_{50}$ was $3.81 \mu \mathrm{g} / \mathrm{ml}$. Representative viruses from major subtypes and recombinant forms could be neutralized by J038, demonstrating that the neutralization breadth of J038 is not biased by particular subtypes (Figure 1D). Neutralization fingerprint analysis indicated that the neutralization profile of J038 clusters with those of the V1V2-targeting antibodies, such as PG9, PGT145 and VRC25.26, suggesting its V1V2- 
specificity.

\section{Cryo-EM structure reveals J038 Fab binds to the V2-Apex}

To determine the structures of J038 or J033 in complex with the HIV-1 Env trimer, we used the neutralization results to select a sensitive strain to make a stabilized trimer (Table S2). The Env from a CRF01_AE strain C1080, which is one of the most sensitive strains to $\mathrm{J038}$ neutralization $\left(\mathrm{IC}_{50}\right.$ of $\left.0.095 \mu \mathrm{g} / \mathrm{ml}\right)$, was stabilized in the prefusion-closed state using the approach described previously (Rawi et al., 2020). To purify the C1080 Env in native buffer condition, we captured the expressed Env onto a protein A column preloaded with a CD4binding site antibody 3BNC117, in which an HRV3C cleavage site was engineered into its hinge region for enzymatic release of the antigen-binding fragment (Fab). We then mixed J038 or J033 Fab with the C1080-3BNC117Fab complex released from the protein A column and further purified for electronic microscopy (EM) studies. Initial negative stain EM indicated that one Fab of J033 or J038 bound to each of the Env apex (Figure S3). Cryo-EM analysis with 3-dimensional reconstruction of the J038-C1080-3BNC117 complex at a nominal resolution of $3.4 \AA$ confirmed a single J038 Fab bound to the V2-apex of the C1080 trimer with one 3BNC117 Fab bound at the CD4-binding site of each protomer (hereafter, we refer protomers as P1, P2 and P3, clockwise when viewing down to the Env apex) (Figures 2A-2B, S4 and Table S3). Unlike some V1V2-specific antibodies like VRC26.25 and PGT145, the heavy chain complementarity-determinant region 3 (CDR H3) of J038 did not insert into the trimer interface at the V2 apex. The interface between antibody J038 and the C1080 Env was well resolved with clear density for glycan N160 extending from 
the surface of Env to interact with the heavy chain CDRs of the J038 Fab. The density of glycan N156 was evident for the portions close to the surface of Env. We also obtained cryo-EM structure for J033, a member of the J038 lineage antibodies. The structure at $4.75 \AA$ resolution showed a very similar binding mode to that of $\mathrm{J038}$ (Figure S4E). We chose the J038 structure that is at higher resolution for further analysis.

Overall, the interface between J038 and C1080 Env trimer buried $1298 \AA^{2}$ surface area from the antibody and $1439 \AA^{2}$ from the HIV-1 Env (Table S4A). The epitope of J038 contained both protein and glycan components, of which $89 \%$ of the surface was from V2 loop of protomer P1 and $11 \%$ of that was from V2 loop of the P2 protomer. Glycans N156 and N160 on strand B of V2 loop on P1 contributed $45 \%$ of the total epitope surface (Figure $2 \mathrm{C}$ and Table S4A). On the antibody side, both heavy chain and light chain were involved in binding of the V2 apex. While heavy chain, which contributed $75 \%$ of the paratope, interacted with protomer $\mathrm{P} 1$ residues and glycans, light chain contacted both protomers P1 and P2 (Figure 2C and Table S4A)

\section{J038 interacts with the V1V2 B-strands on two protomers of the Env}

All CDRs of J038 contributed to binding of C1080 Env (Figure 2C). Unlike the CDR H3 in antibodies such as VRC26.25 and PGT145 which insert into the V2-apex hole on the Env trimer, the 18-residue long CDR H3 of J038 formed extensive anti-parallel $\beta$-strand (main chain) hydrogen bonding interactions with the V2 C-strand of protomer P1 (Figure 3A and Table S4B). Residues at the tip (D99-D100-F100A-Y100C; antibody residues follow Kabat numbering system) of CDR H3 formed hydrogen bonds with side chains of K169, K171 and Y173 
in the same $\mathrm{C}$ strand. In addition, heavy chain residues $\mathrm{Y} 33$ in CDR H1 and R54 in CDR H2 formed hydrogen bonds with D167 and R166 located in the loop between strands $B$ and $C$ of protomer (Figure $3 A$ ). On the light chain side, the CDR L3 tip residues Y91-R92-R93 formed four hydrogen bonds with E164 and K168 on the same loop in protomer P1 (Figure 3A, middle). We also observed hydrogen bonding interactions between J038 light chain residues D1, CDR L1 R27 and CDR L3 R93 and protomer P2 residue N186 located in the loop between V1V2 strands C' and D (Figure 3A, right). Although J038 is a V2Apex bnAb, its EDDY motif mutated into a EDDF motif that interacted with L171 and Y173 not A166 and L169 as other V2-Apex bnAbs (Gorman et al., 2020; Gorman et al., 2016; Lee et al., 2017; McLellan et al., 2011), suggesting that J038 binding to the V2-Apex distinctively.

\section{The binding of J038 to Env is heavily dependent on glycans}

Similar to other V2-Apex bnAbs, J038 interacted with V2-Apex through extensive binding to glycans (Figure 3B). The N160 glycan accounted for the majority $(80 \%)$ of the total glycan contact (Figure 3B and Table S4A). Through the two base GIcNAc, BMA and the Mannose moieties on the D3 branch, glycan N160 interacted with J038 heavy chain by forming 5 hydrogen bonds with N30, R54 and $\mathrm{Y} 100 \mathrm{D}$ in all three CDRs (Figure 3B and Table S4B). These interactions accounted for over one-third (37\%) of the total epitope area. The N156 glycan is responsible for $8 \%$ of the total antibody-Env contact area. Of the two ordered N156 glycan GIcNAc moieties, NAG717 only interacts with D100 in CDR H3 while NAG718 binds to F53 in CDR L2 as well as D100 and F100A in CDR H3. Overall, the glycan contributed nearly half $(45.5 \%)$ of the 
total J038-binding surface, indicating that the glycans plays a very important role in binding of $\mathrm{J} 038$ to V2-Apex.

Viral sequence alignments showed that $\mathrm{C} 1080$ and SHIV 1157 ipd3N4 share the identical sequences on stands B and C of the V2 loop (Figure 4A). This explains why C1080 is potently neutralized by J038 (Table S2). The N160 glycan in SF162 was lost due to the N160K mutation and this rendered SF162 full resistant to the J038 lineage bnAbs. Even though the loop between stands C' and $D$ is slightly divergent between strains in length and residue composition, the four epitope residues (E185, N186, K187 and N189) in this region on protomer P2, which contacted CDR L1 and L3 regions of J038 and provided $11 \%$ of the epitope surface, were relatively conserved (Figure 4A, Table S4B). Overall, the structural information revealed in the J038-C1080 complex provided structural basis for its breadth of neutralization.

To confirm the requirements of key residues and glycans on neutralization, we performed mutagenesis studies with CAP45, a strain potently neutralized by 5 of 6 members of the J038 lineage antibodies (Figure 1C). When the glycosylation site at position 160 was eliminated by either N160A or T162A mutation, CAP45 became fully resistant to neutralization by all five J038 lineage members (Figure 4B). Two other mutations (R166A and K169E) rendered CAP45 more resistant potentially by removing key interactions, such as the stacking effect with Y57 in CDR H2 (Figure 3A and 4B), but the D167N mutation made CAP45 slightly more sensitive. Similar results were observed with the week 350 plasma from G1015R (Figure 4B). Deletion of the N160 glycosylation 
site in two other viruses (25710 and Ce0217) also rendered the viruses full resistant (Fig. S5A). When deglycosylated BG505 trimer was used, the binding of the J038 lineage members were dramatically reduced (Figure 4C and S5B). These results showed that N160 glycan and some key contact sites are required for efficient binding of the J038 lineage antibodies to V2-Apex.

\section{J038 binding induces V2 into a unique "Up" position}

Most V2-Apex bnAbs, such as PG9, VRC26 and PGT145, use their long CDR H3 to penetrate into the apex hole formed at the V2-Apex and interact with the entrance regions of strand $C$ through extensive hydrogen bonding (Gorman et al., 2020; Gorman et al., 2016; Lee et al., 2017; McLellan et al., 2011). In contrast, J038 and J033, which had short CDR H3, bound to the V2apex without penetrating into the apex hole in a similar manner to VRC38 (Figure 5A). Interestingly, when J038 bound to the Env trimer, it induced the V2 strands B and C in protomer P1 into an "up" position (Figure 5B). This unique antibody-induced V2 "up" position has not been observed in any other V2-Apex bnAb/Env complex (Figure 5C). When aligned over the whole Env trimer, the J038-bound V2 strands B and C (residues 154-177) in protomer P1 had an average $\mathrm{C} \alpha$-RMSD of 7.2 between corresponding regions in other antibodybound Env (Figure 5C). In the J038-bound mode, the tip ( $\mathrm{C} \alpha$ of residue 165) of the loop between $B$ and $C$ in protomer P1 moved $11.6 \AA$ to $18.7 \AA$ away from the apex compared to positions in other antibody bound forms (Figure 5D). The strands B and C in the other two gp120 protomers had very similar conformation between the J038- and other antibody-bound Env, for example, that in P2 between J038- and PGT145-bound only had a RMSD of $0.7 \AA$. In summary, the 
J038-induced "up" position of V2 loop represent the first-time observation of such a unique and asymmetric conformation in HIV-1 Env. This unique "up" position of V2-Apex allows easy and tight interaction with CDR3 H3 of J038 (Figure 2C and 5A).

\section{J038 maturation through gradually increased binding areas to glycans}

To understand how the $\mathrm{J} 038$ lineage Abs evolved to more mature overtime, we deduced the unmutated common ancestor (UCA) for both VH and VL sequences and inferred the intermediate antibodies (IAs) (Figures 6A and S1B). There are two clades of the sequences; clade A included all four members (J029, J033, J038 and J040) which are broadly neutralizing, and clade B contained two members (J031 and J044) that neutralized relatively fewer heterologous tier 2 viruses (Figure 1C). To investigate how the gradually accumulated mutations affect neutralization breadth and potency of the J038 lineage Abs, we synthesized and expressed the recombinant UCA and all five IA antibodies. The UCA bound to the heterologous BG505 Env very weakly but gradually gained high affinity as it became more matured (Figure S6). However, like the $\mathrm{CH} 103$ lineage Abs (Liao et al., 2013), the UCA bound the autologous 1157ipd3N4 Env well and the binding affinity of matured J038 was only about 5-fold better than the UCA (Figure S6).

When tested against the same 22-virus panel, the UCA did not neutralize any of the tier 1 and 2 viruses, but it neutralized autologous tier 2 1157ipd3N4 pseudovirus well at $4.97 \mu \mathrm{g} / \mathrm{ml}$ (Figure 6B). The least evolved intermediates IA5 and IA4 were similar and only weakly neutralized one tier 2 virus WTO4160.33 and autologous virus 1157ipd3N4. However, IA5 more potently neutralized the 
autologous virus than UCA while IA4 that led to weakly neutralizing clade B Abs maintained the same low neutralization level as UCA. IA3 and IA2 became more potent by neutralizing $7-8$ strains of tier 2 viruses $(39 \%-44 \%)$ and one of three tier 1 viruses. IA1, which neutralized 12 tier 2 viruses, gained even more neutralization breadth $(70.6 \%)$ similar to that of J038, missing only one virus (BJOX2000) (Figure 6B and 1C). Compared to IA5 and IA4, both IA3 and IA2 became 17-fold more potent to neutralize tier 1 virus MW965.26. However, J038 is 2.3 folds more potent to neutralize autologous virus than IA1. Interestingly, once the lineage Abs gain the ability to neutralize a heterologous tier 2 virus, the level of the neutralization against the virus remain the same during the $\mathrm{Ab}$ maturation process. Taken together, these results demonstrate that the neutralization breadth of the J038 lineage Abs gradually increase as they mature over time.

To understand the relationship between J038 maturation and the development of breadth, we compared the sequences of UCA, intermediate and mature antibodies to identify the somatic hypermutations along with the paratope residues of $\mathrm{J038}$ (Figure 6C). We also performed template-based homology modeling of the UCA and intermediate antibodies using the J038C1080 structure (Figure 6D). Mapping of the paratope indicated that 11 and 6 of the paratope residue were directly encoded by the VH and VL genes of UCA, respectively, which provided $627 \AA^{2}$ binding area (Figures $6 \mathrm{C}-6 \mathrm{D}$ and Table S5A). In IA5, four and three residue substitutions in VH and VL, respectively, evolved at the contact sites and added an additional binding area of $333 \AA^{2}$. IA3 gained another 4 and 2 residue substitutions in $\mathrm{VH}$ and $\mathrm{VL}$, respectively, at the contact sites, and the binding area was increased by another $167 \AA^{2}$. When 
there was only one residue substitution was found in $\mathrm{VH}$ or $\mathrm{VL}$ region of IA2 and both together further increased binding area by $95 \AA^{2}$. The additional mutations in IA1 and mature Abs did not directly alter the binding area (Figures 6C-6D and Table S5B).

We observed that paratope residues from UCA primarily provided core interactions to stand $\mathrm{C}$ of protomer P1 (Figure 6D). The early increased binding area by IA5 further strengthened the contact with protein components of V2 strand $\mathrm{C}$. It is interesting to noticed that the later increased binding area by IA3 and IA2 were at the peripheral of the paratope and were mainly (6 of 10 residue substitutions) for binding of the N156 and N160 glycans. These results demonstrate that the J038 lineage Abs matured by strengthening interactions to the $\mathrm{V} 2$ residues and then increasing their binding areas to glycans on Env to broaden the neutralization breadth (Figure 6D).

We also performed Biolayer interferometry (BLI) assay to determine the binding kinetics of inferred antibodies (Figure S6). The affinity of UCA and IAs to autologous gp120 was consistent with their neutralizing potency against the autologous pseudovirus. The BLI assay also showed that IA4, the progenitor for clade B Abs in the phylogenetic tree, bound to HIV-1 Envs much weaker than IA5, similar as UCA. J031 and J044 were evolved from IA4 and both had a narrower neutralization breadth (Figure 1D). These results indicate that the mutations accumulated in IA4 had a negative influence on antibody evolution towards broad neutralization.

\section{Limited mutations at the Ab-Ag interface in the env gene}

A small region (positions 156-188) including two glycosylation sites (N156 
and $\mathrm{N} 160$ ) in strands $\mathrm{B}$ and $\mathrm{C}$ of $\mathrm{V} 2$ was responsible for the contact with J038 (Figure 3). Examination of viral sequences at this region from the longitudinal plasma samples in G1015R showed no mutations at week 27. No plasma samples from the next 187 weeks were available for sequence analysis. By week 214 , all 23 viral sequences had the $1165 \mathrm{~L}$ and $\mathrm{K} 171 \mathrm{R}$ mutations, while the K168R and V172A mutations accounted for $69 \%$ and $92 \%$ of the virus population, respectively (Figure 7A). All three mutations (I165L, K171R and V172A) are fixed in the viral population by week 350 while the K168R mutation became undetectable. Four residues in the V2 strands C' and D from protomer $\mathrm{P} 2$ in the Env trimer were found to contact J038 (Figure 4A). However, no mutations in this region were detected by week 214 (Figure 7A). A few different mutation combinations were found in some sequences by week 350 . Interestingly, no mutations that can eliminate N156 and/or N160 glycosylation sites were found throughout the 7-year follow-up period.

To understand how these mutations affected viral susceptibility to neutralization by the $\mathrm{J} 038$ lineage antibodies, we introduced these three mutations (I165L, K171R and V172A) individually or all together into the autologous 1157ipd3N4 env gene. Since both the I165L and K171R were the Env-J038 contact sites, the I165L and K171R mutants were 7 and 5 folds more resistant, respectively, to the J038 lineage Abs (Figure 7B). The V172A mutation was not a contact site and the V172A mutant was only slightly more resistant to neutralization (3 folds) than the wild type 1157ipd3N4. However, when the I165L, K171R and V172A mutations (Trimut) were tested together, it was about 20-fold more resistant. We also tested the I192T mutation which is not at the Env-J038 contact site but fixed in viral population by week 214 and 
350. The I192T mutant had similar neutralization susceptibility as the wild type 1157ipd3N4.

Structure modelling of the $1165 \mathrm{~L}$ and $\mathrm{K} 171 \mathrm{R}$ mutations at the contact sites showed that both mutations might potentially cause clashes with CDR L3 and CDR H3, respectively (Figure 7C). This explained why both mutations alone modestly increased resistant to neutralization by the J038 lineage antibodies. Although V172A is not involved in antibody binding, but all I165L, K171R and V172A mutations together could have a collective unfavorable interaction with J038 (Figure 7C). These results demonstrate that over seven years of infection, a few mutations accumulated at the J038 contact sites in the env gene and only modestly impacted viral susceptibility to neutralization by the J038 lineage Abs in G1015R.

\section{Discussion}

We sorted single memory B cells from SHIV-infected Chinese rhesus macaques using HIV-1 Env V2-Apex differential baits and obtained bnAbs that can broadly neutralize $54 \%$ of the 208 HIV-1 strains. This is the first case to show that bnAbs can be successfully obtained from monkeys that developed broad neutralization activity in sera after seven years of infection. This demonstrates that the bnAbs can be elicited in SHIV infected macaques after a long period of infection time as determined by longitudinal plasma samples and reconstructed Abs evolved from UCA to mature bnAbs. This lineage of bnAbs targets V2-Apex in the HIV-1 Env trimer. However, sequence, structure and phenotype analyses of the J038 lineage bnAbs show a number of unique features that can have important implications in future HIV vaccine design. 
The six J038 linage bnAbs are the only predominant gene family (14\%) among 44 sorted B cells. None of other 38 Abs share the same VH and VL sequence from the same gene family. The J038 lineage bnAbs were derived from VH4 gene family, which is dominant usage in rhesus macaque B cell repertories (Dai et al., 2015; Sundling et al., 2012a). A number of nAbs obtained from macaques are found to belong to the VH4 gene family (Dai et al., 2015; Han et al., 2019; Kong et al., 2019; Sundling et al., 2012a; Wang et al., 2017). These results indicate that the $\mathrm{VH} 4$ gene family may be preferred for bnAbs, regardless any binding specificities, in SHIV infected or vaccinated macaques. Similar VH germline orthologous gene is also found in humans (Figure S7), indicating that such Abs are likely to be induced in humans too.

Most of previous V2-apex targeting bnAbs obtained from HIV-1 infected humans have a long anionic CDR H3 and high SHM except VRC38 (Andrabi et al., 2015; Cale et al., 2017; Gorman et al., 2016; Moore et al., 2017). However, the CDR H3 of the J038 lineage bnAbs, like VRC38, are short, with only 18 amino acids. Like other V2-apex bnAbs (Gorman et al., 2020; Gorman et al., 2016; Lee et al., 2017; McLellan et al., 2011), only one J038 Fab binds to the Env trimer. However, binding of J038 induces a unique "up" conformation of V2 in the main binding protomer, which provides $\sim 90 \%$ of the epitope surface, while the other two V2 loops in the same Env trimer remain unchanged. J038 binding to Env is heavily dependent on two glycans (N156 and N160), accounting for $45 \%$ of the total contact area. The deletion of either of these glycosylation sites in heterologous env genes results in much reduced binding and renders the viruses resistant to neutralization. The "up" position of V2 induced by J038 represents the first observation of such conformation in HIV-1 Env not identified 
in other V2-apex bnAb-bound Envs (Cale et al., 2017; Gorman et al., 2020; Lee et al., 2017; Walker et al., 2009). This unique binding pattern of J038 may facilitate tighter interaction with $\mathrm{J} 038$ and thereby enables binding to monomeric gp120.

Compared to the other bnAbs with V2 or other specificities (Klein et al., 2013; Kwong et al., 2013; Sok and Burton, 2018), the J038 linage bnAbs does not have strong selection pressure on the viral populations during 7 years of infection. Only three mutations (I165L, K171R and V172A) at the Ab-Env contact sites were strongly selected by week 350 post infection. Among them, the $1165 \mathrm{~L}$ and K171R mutations interact with $\mathrm{J} 038$ and either mutation rendered the virus slightly more resistant to neutralization by J038. No mutations that affect either N156 or N160 glycosylation sites were detected. This suggests that the maturation of neutralization breadth in $\mathrm{G} 1015 \mathrm{R}$ may not require extensive mutations at the contact sites.

To increase the neutralization breath, the $\mathrm{CH} 235$ lineage bnAbs decrease the binding area size to focus on only the highly conserved CD4 binding site core on Env (Bonsignori et al., 2016) while the $\mathrm{CH} 103$ lineage bnAbs change the angle of $\mathrm{VL}$ to more likely accommodate the size changes of the V5 loop during their maturation process (Fera et al., 2014). The J038 lineage bnAbs gain its neutralization breadth through a different mechanism. On the core binding area contacted by UCA-encoded residues, the mutations in more evolved intermediary IA5 increased $54 \%$ of the binding area by mutations in both $\mathrm{VH}$ and $\mathrm{VL}$ chains. More mutations accumulated in IA3 and IA2 further increased the binding by another $42 \%$ binding area. The increased binding area between $\mathrm{Ab}$ and Env during the maturation process of the J038 lineage bnAbs 
allow them to bind Env at higher affinity. Importantly, the majority increased binding from UCA is through binding to both N156 and N160 glycans (mainly N160 glycan).

Our previous studies showed that the UCA of bnAbs do not neutralize T/F viruses or heterologous viruses that initiated the bnAb lineage (Bonsignori et al., 2017; Gao et al., 2014; Liao et al., 2013). However, the UCA of DH727 obtained from a vaccinated macaque could neutralize a heterologous CNE8 (Han et al., 2019), and the UCA of CAP256-VRC26 could neutralize CAP256SU weakly (Doria-Rose et al., 2014; Gorman et al., 2016). Here we found that the UCA of J038 lineage antibodies moderately neutralize autologous 1157ipd3N4 virus, but not any other tier 1 and tier 2 viruses. This demonstrate that the germline of some bnAbs is fully capable to neutralize autologous $\mathrm{T} / \mathrm{F}$ viruses. Thus, bnAbs derived from such germlines may be easier to induce by vaccines than those bnAbs derived from germlines that cannot neutralize the autologous T/F viruses that trigger the lineages.

When the J038 lineage bnAbs continue to evolve, they become more potent to neutralize the autologous virus and gain the ability to neutralize more heterologous tier 2 viruses. Since N156 and N160 glycans are conserved among global HIV-1 strains and both contribute nearly $50 \%$ of the Ab binding area, the increasing binding area to both glycans may lead to broad neutralization of diverse HIV-1 strains. Importantly, the invariant of both glycans during 7 years of infection allows the J038 lineage bnAbs to continuously gain additional binding area to them during their maturation process. 
In summary, mature $\mathrm{J} 038$ is broadly neutralizing ( $54 \%$ of HIV-1 stains), the J038 lineage is the predominant V2-binding Abs in G1015R, the UCA of the J038 lineage bnAbs can neutralize the autologous virus, it binds a unique "up" V2 position, and its maturation heavily depends on glycans. Moreover, J038 has a relatively short HCDR3 and relatively low SHM rate ( $20 \%)$. Finally, its maturation does not drive extensive mutations in the Ab-Env contacts sites. All these together indicate that such bnAbs may be easier to induce. This has important implications in HIV vaccine design to induce J038 like bnAbs in humans.

Limitations of the Study: Broadly neutralizing antibodies with only V2 specificity were characterized using the HIV-1 Env V2-Apex differential baits in this study. This may not fully represent all the bnAb activities, since nAbs with CD4bs and V3 specificities were also identified in the same macaque. HIV-1 Env specific memory B cells were sorted and characterized from only one macaque. Studying additional macaques infected with SHIV 1157 ipd3N4 can further strengthen our findings. Since this study was carried out with the previously archived blood samples, many samples were exhausted for our previous studies. We did not have plasma samples available from week 27 through week 214 to study if viral mutations during this long infection period have any impacts on viral escape from the J038 lineage bnAbs. Since J038 sequence is very similar to its UCA and IA sequences and directly evolved from them, we used the template-based homology modeling to determine the structure basis for the J038 maturation process. Resolving cryo-EM structures between the HIV-1 Env 
and the UCA and IAs of the J038 lineage bnAbs could reveal structure details in higher resolution.

\section{Acknowledgments}

We thank M. Anthony Moody, Kevin O. Saunders and Barton F. Haynes for providing V2 differential baits and rhesus macaque antibody expression vectors. We also thank members of the Structural Biology Section, Vaccine Research Center, for discussions and comments on the manuscript. This project was supported by the National Natural Science Foundation of China (Grant No. 31970888 and No. 82002138), Key Projects in the National Science \& Technology Pillar Program in the Thirteenth Five-year Plan Period (Grant No. 2018ZX10731101-001-010 and 2018ZX10731101-002-003), Program for JLU Science and Technology Innovative Research Team (grant no. 2017TD-05). This research was, in part, supported by the Intramural Research Program of the Vaccine Research Center, National Institute of Allergy and Infectious Diseases (NIAID) and the National Cancer Institute's National Cryo-EM Facility at the Frederick National Laboratory for Cancer Research under contract HSSN261200800001E.

\section{Author contributions}

Conceptualization, N.G., C.Q., T.Z., X.Y. and F.G.; Data acquisition, N.G., Y.G., L.M., C.W., W.W., X.L., T.G., M.L., N.D.; Data analysis, N.G., C.W., M.L., N.D., B.Y., H.L., C.Q., P.D.K., J.R.M., T.Z., X.Y., F.G.; Bioinformatic analysis, K.W., T.B.K., J.L.; Monkey experiment, W.W., C.Q., H.N.; Neutralization 
fingerprint analysis, A.J., Y.T.; Design and generation of HIV-1 Env proteins, J.G., A.S.O., R.R., P.K.; Cryo-EM analysis, N.D., W.W., C.S., K.H.; Structure analysis, T.Z., P.D.K.; Writing - Original Draft, N.G., Y.G.; Writing - Review \& Editing, N.G., X.Y., T.Z., F.G.; Supervision, F.G.; Funding acquisition, C.W., X.Y., F.G

\section{Declaration of interests}

The authors declare no competing interests.

\section{Figure legends}

\section{Supplemental Materials}

\section{STAR $\star$ METHODS}

- KEY RESOURCES TABLE

- CONTACT FOR REAGENT AND RESOURCE SHARING

- EXPERIMENTAL MODEL AND SUBJECT DETAILS

○ Cell lines

- Macaque PBMC samples

- METHOD DETAILS

- Epitope-specific single B cell sorting

- Antibody sequence analysis

- Expression of recombinant antibodies

- Site-directed mutagenesis

- Generation and titration of HIV-1 Env pseudoviruses

- Neutralizing Assay

- Expression and purification of HIV-1 Env proteins

- Enzyme-linked immunosorbent assay

- Biolayer interferometry (BLI) measurements

- Inference of unmutated and intermediate evolution antibodies

- Preparation of antibody Fab region and antibody-Env complexes

- Cryo-EM grid preparation and data collection

- Cryo-EM data processing and refinement

- Structural analysis

- QUANTIFICATION AND STATISTICAL ANALYSIS

- DATA AND CODE AVAILABILITY 
KEY RESOURCES TABLE

\section{RESOURCE AVAILABILITY}

\section{Lead Contact}

Further information and requests for resources and reagents should be directed to and will be fulfilled by Feng Gao (fgao@duke.edu).

\section{Materials Availability}

Expression plasmids generated in this study for expressing antibodies and Env mutants will be shared upon request.

\section{EXPERIMENTAL MODEL AND SUBJECT DETAILS}

\section{Cell lines}

HEK 293T Cells (Cat\# CRL-3216) were from ATCC. Expi293F ${ }^{\mathrm{TM}}$ Cells (Cat\# A39240) were from Thermo Fisher Scientific. TZM-bl Cells were from NIH AIDS reagent program. 293-6E cells were from NTCC.

\section{Macaque PBMC samples}

Peripheral blood mononuclear cells (PBMCs) were collected from the Chinese rhesus macaques $\mathrm{G} 1015 \mathrm{R}$ infected with $\mathrm{SHIV}_{1157 \mathrm{ipd} 3 \mathrm{~N} 4}$ via the intrarectal route (Gao et al., 2018). Monoclonal antibodies were isolated from the PBMCs collected at week 350 when broad neutralization activity was detected and the viral load was maintained at a high level. 


\section{METHOD DETAILS}

\section{Epitope-specific single B cell sorting}

Single B cell sorting was performed as described previously (Wiehe et al., 2014; Zhang et al., 2016). Frozen PBMCs $\left(\sim 1 \times 10^{7}\right.$ cells $)$ were thawed in RPMI 1640 with 10\% FBS and washed with PBS with 5\% FBS. Multicolor staining was performed using a panel of fluorophore-labeled antibodies (CD3-PECF594, CD16-PE-Cy5, CD14-PE-Cy7, CD20-BB515, IgD-PE, CD27-APC-Cy7, AmCyan-DEAD, wild type gp120-BV421, mutant gp120-AF647). All staining was followed by washing and resuspension with PBS with $5 \%$ FBS. Single epitope-specific cells were sorted into individual wells in 96-well PCR plates. Each well contains $20 \mu \mathrm{L}$ lysis buffer consisting of $0.5 \mathrm{U}$ RNase OUT (Invitrogen, Grand Island, NY), $0.0625 \mu \mathrm{L}$ Igepal (Sigma, St. Louis, MO), $5 \mu \mathrm{L} 5 \mathrm{x}$ SuperScript III First-Strand Buffer and $1.25 \mu \mathrm{L}$ DTT provided with the Superscript III Reverse Transcriptase kit (Invitrogen, Grand Island, NY). The PCR plates containing lysed single cells were stored at $-80 \mathrm{C}^{\circ}$. Sorting data was analyzed with FlowJo software (FlowJo, Treestar, Ashland, OR).

\section{Antibody sequence analysis}

Single cell RT-PCR for amplification of IgG heavy and light chain genes was performed as described previously (Sundling et al., 2012b; Wiehe et al., 2014). The cDNA of Ab mRNA was synthesized with 150 ng Random Hexamers 
(Qiagen, Germantown, MD), 200 U Superscript III and $1 \mathrm{mM}$ dNTPs by incubating at $42^{\circ} \mathrm{C}$ for 10 minutes, $25^{\circ} \mathrm{C}$ for 10 minutes, $50^{\circ} \mathrm{C}$ for 60 minutes and $94^{\circ} \mathrm{C}$ for 6 minutes. The heavy and light chains variable regions were amplified separately from the cDNA templates with two rounds of PCR using panels of specific primers to distinguish different Ab gene families. In the first round PCR, $3.5 \mu \mathrm{L}$ of $\mathrm{cDNA}, 5 \mu \mathrm{L} 10 \times$ High Fidelity PCR Buffer, $1 \mu \mathrm{L} 10 \mathrm{mM}$ dNTPs, $2 \mu \mathrm{L}$ 50mM MgSO4, $1 \mu \mathrm{L}$ 5'-primer mix and $1 \mu \mathrm{L} 25 \mu \mathrm{M}$ of 3'-primer, $0.25 \mu \mathrm{L}$ High Fidelity Taq (Invitrogen, Grand Island, NY) were added into a 50 $\mu \mathrm{L}$ reaction. The PCR thermocycling conditions were as follows: one cycle at $94^{\circ} \mathrm{C}$ for 2 minutes; 35 cycles of a denaturing step at $94^{\circ} \mathrm{C}$ for 15 seconds, an annealing step at $64^{\circ} \mathrm{C}(\lg \mathrm{K}$ and $\lg \lambda)$ or $62^{\circ} \mathrm{C}(\operatorname{lgH})$ for $30 \mathrm{sec}$, and an extension step at $68^{\circ} \mathrm{C}$ for 1 minute; and one cycle of an additional extension step at $68^{\circ} \mathrm{C}$ for 5 minutes. The second round PCR was performed with $1 \mu \mathrm{L}$ of the first round PCR product, $5 \mu \mathrm{L}$ 10× PCR Buffer, $1 \mu \mathrm{L} 10 \mathrm{mM}$ dNTPs, $1 \mu \mathrm{L}$ Jumpstart Taq (Sigma, St. Louis, MO) and $1 \mu \mathrm{L} 25 \mu \mathrm{M}$ of $\lg \mathrm{H}$, $\lg \mathrm{K}$, or $\lg \lambda$ inner forward and reverse variable region primers. The second round PCR thermocycling conditions were as follows: one cycle at $94^{\circ} \mathrm{C}$ for 5 minutes; 35 cycles of a denaturing step at $94^{\circ} \mathrm{C}$ for 30 seconds, an annealing step at $64^{\circ} \mathrm{C}(\lg \mathrm{k}$ and $\lg \lambda)$ or $62^{\circ} \mathrm{C}(\operatorname{lgH})$ for $30 \mathrm{sec}$, and an extension step at $72^{\circ} \mathrm{C}$ for 1 minute; and one cycle of an additional extension step at $72^{\circ} \mathrm{C}$ for 10 minutes. PCR products with correct sizes were directly sequenced. Alignments of sequences to germline $\mathrm{V}$, (D), and J genes were performed using IMGT/V-QUEST (www.imgt.org). 


\section{Expression of recombinant antibodies}

Linear Ab expression cassettes were generated with paired heavy and light chain PCR products from the same single cells by adding a CMV promoter to each Ab chain to express recombinant Abs (Liao et al., 2009). When the Abs were found to bind autologous SHIV $_{1157 i p d 3 N 4}$ gp120, both heavy and light chains of the Abs were cloned into the pCDNA3.1+ expression vector for large scale expression. The complete recombinant IgG proteins were expressed by cotransfection of paired heavy and light chain expressing plasmids into Expi293F (Thermo Fisher, Waltham, MA) according to the protocol for the ExpiFectamine $^{\mathrm{TM}} 293$ Transfection Kit. Following 4-6 days incubation, culture supernatants were filtered and recombinant Abs were purified with Protein $A$ Agarose (Thermo Pierce, Waltham, MA). Abs were eluted with IgG Elution Buffer (Thermo Pierce, Waltham, MA) and collected in $1 \mathrm{M}$ Tris $\mathrm{pH} 8$ solution. The antibody solution was exchanged against PBS and the purified Abs were stored at $-80^{\circ} \mathrm{C}$.

\section{Site-directed mutagenesis}

Mutations in the SHIV ${ }_{1157 i p d 3 N 4}$ env gene were introduced by Site-Directed mutagenesis PCR (Gao et al., 2020). Briefly, a pair of oligonucleotide primers containing the desired mutation were used for PCR amplification using KODplus DNA polymerase (TOYOBO, Osaka). After amplification, the parental 
plasmid DNA template in the mixture was digested by Dpn I (Takara, Kusatsu,

Shiga) treatment and the products with mutations were remained. Finally, the PCR products were transformed into DH5a competent cells and all env mutant plasmids were confirmed by sequencing.

\section{Generation and titration of HIV-1 Env pseudoviruses}

The Env pseudoviruses were generated by co-transfecting $8 \mu \mathrm{g}$ of the env expression plasmid and $16 \mu \mathrm{g}$ of the env-deficient HIV-1 backbone (pSG3 $\Delta \mathrm{Env}$ ) in to 293 T cells using Lipofectamine 2000 transfection reagent (Invitrogen, Grand Island, NY) in T75 flask according to the manufacturer's protocol. Cell culture supernatants were harvested $48-72 \mathrm{~h}$ following transfection and the subpackaged virus stock were stored at $-80^{\circ} \mathrm{C}$. The pseudoviruses were titrated by 115 -fold serial dilution of each virus stock used to infect $1 \times 10^{4} \mathrm{TZM}$ bl cells DMEM containing $10 \mu \mathrm{g} / \mathrm{mL}$ of DEAE-dextran, and a dilution which gave a relative luminescence units (RLU) signal that around 100 fold above average cell only wells was chosen to apply in neutralizing assay.

\section{Neutralizing Assay}

Neutralization activities of the antibodies were detected on TZM-bl cells as previously described (Gao et al., 2018). Briefly, purified antibodies (50 $\mu \mathrm{g} / \mathrm{ml})$ were serially diluted at a 1:3 ratio with DMEM. After incubation with certain concentration of Env-pseudoviruses at $37^{\circ} \mathrm{C}$ for $1 \mathrm{~h}$, the $1 \times 10^{4} \mathrm{TZM}-\mathrm{bl}$ cells in 
DMEM containing $10 \mu \mathrm{g} / \mathrm{mL}$ of DEAE-dextran were added into each well to be infected. After 48-72 h incubation, $200 \mu \mathrm{L}$ of supernatant was removed and 40 $\mu \mathrm{L}$ of luciferase substrate was added into each well to test the RLU. The $50 \%$ inhibitory concentrations $\left(\mathrm{IC}_{50}\right)$ were defined as antibodies concentration at which RLU were reduced by $50 \%$ compared with average RLU of virus control after subtraction of background RLU of cell control. A response was considered positive for neutralization if the $\mathrm{IC}_{50}$ concentration was lower than $50 \mu \mathrm{g} / \mathrm{mL}$.

The further neutralizing breadth and potency of J038 was evaluated on a panel of 208 circulating HIV-1 strains with microneutralization assay as previously described performed with an automated workstation (Doria-Rose et al., 2013; Sarzotti-Kelsoe et al., 2014) and the $\mathrm{IC}_{50} / \mathrm{IC}_{80}$ was determined.

\section{Expression and purification of HIV-1 Env proteins}

The gp120 monomer and BG505 UFO trimer (Kong et al., 2016) were produced by transient transfection of plasmids into 293-6E suspension cells (NRC, Ottawa, ON) in serum-free medium, using a high-density transfection protocol. Briefly, 293-6E cells were thawed and incubated with OPM-293 CD05 Medium (OPM Biosciences, Shanghai) in a shaker incubator at $37^{\circ} \mathrm{C}$, with 100 rpm. and $5 \% \mathrm{CO}_{2}$. The transfection procedure has been previously described (Sellhorn et al., 2009). Briefly, $0.25 \mathrm{mg}$ of Env plasmid in $2.5 \mathrm{~mL}$ of OPM-293 CD05 Medium was mixed with $1 \mathrm{~mL}$ of PEI $(1.0 \mathrm{mg} / \mathrm{mL})$ in $2.5 \mathrm{~mL}$ of OPM-293 CD05 Medium. After incubation for $15 \mathrm{~min}$, the DNA-PEI complex was directly 
added to $200 \mathrm{~mL}$ 293-6E cells. Culture supernatants were collected 3 days after transfection, then clarified by centrifugation at 3,000 rpm for $1 \mathrm{~h}$ and filtered using $0.45-\mu \mathrm{m}$ filters (Thermo Scientific, Waltham, MA). The Env proteins were extracted from the supernatants using Lentil Lectin Sepharose ${ }^{T M}$ 4B (GE Healthcare, Boston, MA). The bound proteins were eluted with PBS containing $500 \mathrm{mM}$ methyl-a-D-mannopyranoside and then concentrated for use. The affinity-purified Env proteins were further purified by size exclusion chromatography (SEC) using a Superdex 200 Increase 10/300 GL column (GE Healthcare, Boston, MA).

The HIV-1 C1080 Env for structural studies was expressed by transient transfection in 293 Freestyle cells. Briefly, $1 \mathrm{mg}$ of DNA was transfected into $1 \mathrm{~L}$ of cells using Turbo293 transfection reagent, and the cells were allowed to grow at $37^{\circ} \mathrm{C}$ for 6 days. Following expression, the supernatant was cleared by centrifugation and filtration, Supernatant was passed through a protein $A$ column preloaded with CD4-binding site antibody 3BNC117, which has been engineered to have an HRV3C cleavage site at the hinge region. The resin was washed with PBS until the absorbance at $280 \mathrm{~nm}$ reached 0 and resuspended with 5-10 ml PBS. $200 \mu \mathrm{l}$ of HRV-3C protease was then added to the resin mixture and incubated at $4{ }^{\circ} \mathrm{C}$ overnight to release the Env-3BNC117 Fab complex. Column flow-through and PBS wash (3 column volumes) were collected, concentrated and further purified with a Superdex 200 Increase 10/300 column to remove excess 3BNC117 Fab and HRV3C protease. 


\section{Enzyme-linked immunosorbent assay}

Briefly, the autologous gp120 monomer or BG505 UFO trimer was coated into plates with $200 \mathrm{ng} /$ well overnight at $4^{\circ} \mathrm{C}$. Plates were washed three times with PBST (0.1\% Tween 20 in PBS) and then blocked by PBS with 3\% BSA for $2 \mathrm{~h}$ at $37^{\circ} \mathrm{C}$. Then 5 -fold serially diluted antibodies from $50 \mu \mathrm{g} / \mathrm{mL}$ were added into the wells with $1 \% \mathrm{BSA}$ in PBS for $1 \mathrm{~h}$ incubation at $37^{\circ} \mathrm{C}$. Plates were washed 3 times with PBST and horseradish peroxidase (HRP) rabbit antimonkey lgG antibody (Bioss, Beijing) were added at 1: 1000 dilution for $1 \mathrm{~h}$ at $37^{\circ} \mathrm{C} .100 \mu \mathrm{L} /$ well tetramethylbenzidine (TMB) substrate were added and incubated for $15 \mathrm{~min}$ at room temperature in the dark and the reaction was stopped by adding $50 \mathrm{uL}$ of $2 \mathrm{M} \mathrm{H}_{2} \mathrm{SO}_{4}$. The readout was detected at a wavelength of $450 \mathrm{~nm}$.

\section{Biolayer interferometry (BLI) measurements}

BLI experiments were performed using the OCTET Red96 system (FORTÉBIO, San Jose, CA ) to determine binding curves of monoclonal antibodies for BG505 UFO and autologous gp120 (Sok et al., 2014). Briefly, 10 $\mathrm{ng} / \mathrm{mL}$ monoclonal antibodies were immobilized onto Anti-hlgG-Fc Capture (AHC) biosensors (FORTÉBIO, San Jose, CA ) . Six serial dilutions from 800 $\mathrm{nM}$ to $25 \mathrm{nM}$ of each antigen were prepared and flowed as analyte in solution. The binding experiment was performed at $30^{\circ} \mathrm{C}$ using the following protocol: 
baseline 1 (300 s), load antibodies (300 s), baseline 2 (60 s), Ab-antigen association (300 s) and dissociation (300 s). Binding affinity constants were determined using OCTET software Data Analysis HT 9.0 (FORTÉBIO, San Jose, CA ).

\section{Inference of unmutated and intermediate evolution antibodies.}

The clonal tree, ancestral intermediate sequences, and unmutated common ancestor (UCA) sequence for the JO31 clone were inferred using Cloanalyst paired heavy and light chain UCA inference and clonal tree reconstruction (Kepler, 2013).

\section{Preparation of the antibody Fab fragment and antibody-Env complex}

For production of the Fab fragments of J033 and J038, a HRV3C protease cleavage site was inserted at the hinge region of $\lg G$ heavy chain gene as previously described (Shen et al., 2020). Cell culture supernatants of J033 and J038 were harvested, filtered, and loaded on Protein A columns (GE Health Science). Antibodies were eluted with IgG elution buffer (Pierce) and immediately neutralized by addition of one tenth volume of $1 \mathrm{M}$ Tris- $\mathrm{HCl} \mathrm{pH}$ 8.0. To generate Fab fragments, purified IgGs were digested with HRV3C overnight at $4^{\circ} \mathrm{C}$. The mixtures were passed through protein $\mathrm{A}$ columns again to remove Fc fragment or undigested IgG and the flow-though, which contained antibody Fab, were collected and concentrated for preparation of antibody-Env complex. 
To obtain the J038 or J033 Fab-Env complexes, purified Fab was mixed with the C1080-3BNC117 complex at a 3.6:1 molar ratio and passed through a Superdex 200 Increase 10/300 column to separate excess Fab and the J038or J033-C1080-3BNC117 complex.

\section{Cryo-EM grid preparation and data collection}

A sample of the J038-Env-3BNC117 or J033-Env-3BNC117 complexes at $1 \mathrm{mg} / \mathrm{ml}$ was diluted $1 \mathrm{mg} / \mathrm{ml}, 0.5 \mathrm{mg} / \mathrm{ml}$, and $0.25 \mathrm{mg} / \mathrm{ml}$ in PBS buffer. $3 \mu \mathrm{l}$ of each sample applied to a glow discharged Quantifoil R 1.2/1.3 Cu 200 mesh grid (Glow discharger settings: 0.30 mbar, 15 mAMP, and 5 seconds). Grids were then plunge frozen in liquid ethane with an FEI vitrobot and stored in liquid nitrogen (Vitrobot settings: $4{ }^{\circ} \mathrm{C}$ temperature, $100 \%$ humidity, blot time 2 seconds, blot force 0 , wait time 5 seconds, blot total 1 , drain time 0 ).

\section{Cryo-EM data processing and refinement}

Images of the J033-Env-3BNC117 or J038-Env-3BNC117 complexes were collected on Titan Krios at magnification 18k with super-resolution mode. The original sampling is $0.68 \AA$ per pixel. We used bin=2 when processing the data and the sampling is $1.36 \AA ̊$ per pixel.

The workflow on both data sets is shown in Figure Sxx. Relion 3.08 was employed to process the data. The images were further downscaled for the preprocessing process: manual particle picking, 2D classification to generate 2D templates for later auto particle picking, and 3D classification to generate a 
low-resolution model. Then the templates were applied to original data to do auto particle picking. After following the workflow of Relion, we got $3.4 \AA$ structure without using CTF Refinement and Bayesian Polishing to further push the resolution. Correlations between cryo-EM maps and atomic models were assessed using phenix.mtriage (Afonine et al., 2018). UCSF Chimera was used for docking and visualization (Pettersen et al., 2004). The coordinates of HIV-1 BG505 SOSIP in complex with 3BNC117 complex (PDB 5V8L) was used as initial models for fitting the cryo-EM map. The initial model for J038 Fab variable region was obtained using the SAbPred server (Shen et al., 2020). The J033 Fab was modelled based on refined J038 coordinates. Iterative model building and real space refinement were carried out using Coot (Emsley and Cowtan, 2004) and Phenix to fit the coordinates to the electron density map. Molprobity (Davis et al., 2004) was used to validate geometry and check structure quality.

\section{QUANTIFICATION AND STATISTICAL ANALYSIS}

All inhibitory values $\left(\mathrm{IC}_{50}\right.$ and $\left.\mathrm{IC}_{80}\right)$ of antibodies were calculated with a formally validated Excel-based macro (Piehler et al., 2011). The phylogenetic tree of neutralization results for 208-panel was constructed with Clustal X2. The ELISA binding curves were fitted using GraphPad Prism 8. The affinity constants of BLI were determined using OCTET software Data Analysis HT 9.0. The sequence alignments of antibodies or env coding regions were processed with Seaview 5.0.1. Cryo-EM data were processed and analyzed using 
cryoSPARC and Relion. Cryo-EM structural statistics analysis were carried out using Phenix, Molprobity, EMringer, PDBePISA and Chimera.

\section{DATA AND CODE AVAILABILITY}

The sequences of 44 pairs of isolated antibodies and inferred intermediates have been deposited in Genbank, the accession numbers are: MZ234477MZ234576. Cryo-EM map and coordinates of the J033-HIV-1 Env complex has been deposited under the accession codes EMDB:EMD-24128, PDB:7N28; Cryo-EM map and coordinates of the J038-HIV-1 Env complex has been deposited under the accession codes EMDB:EMD-24071, PDB:7MXD.

\section{References}

Adams, P.D., Afonine, P.V., Bunkoczi, G., Chen, V.B., Davis, I.W., Echols, N., Headd, J.J., Hung, L.W., Kapral, G.J., Grosse-Kunstleve, R.W., et al. (2010). PHENIX: a comprehensive Python-based system for macromolecular structure solution. Acta Crystallogr D Biol Crystallogr 66, 213-221.

Afonine, P.V., Klaholz, B.P., Moriarty, N.W., Poon, B.K., Sobolev, O.V., Terwilliger, T.C., Adams, P.D., and Urzhumtsev, A. (2018). New tools for the analysis and validation of cryo-EM maps and atomic models. Acta Crystallogr D Struct Biol 74, 814-840.

Alam, S.M., Aussedat, B., Vohra, Y., Meyerhoff, R.R., Cale, E.M., Walkowicz, W.E., Radakovich, N.A., Anasti, K., Armand, L., Parks, R., et al. (2017). Mimicry of an HIV broadly neutralizing antibody epitope with a synthetic glycopeptide. Sci Transl Med 9. 
Andrabi, R., Bhiman, J.N., and Burton, D.R. (2018). Strategies for a multi-stage neutralizing antibody-based HIV vaccine. Curr Opin Immunol 53, 143-151. Andrabi, R., Voss, J.E., Liang, C.H., Briney, B., McCoy, L.E., Wu, C.Y., Wong, C.H., Poignard, P., and Burton, D.R. (2015). Identification of Common Features in Prototype Broadly Neutralizing Antibodies to HIV Envelope V2 Apex to Facilitate Vaccine Design. Immunity 43, 959-973.

Bonsignori, M., Kreider, E.F., Fera, D., Meyerhoff, R.R., Bradley, T., Wiehe, K., Alam, S.M., Aussedat, B., Walkowicz, W.E., Hwang, K.K., et al. (2017). Staged induction of HIV-1 glycan-dependent broadly neutralizing antibodies. Sci Transl Med 9.

Bonsignori, M., Zhou, T., Sheng, Z., Chen, L., Gao, F., Joyce, M.G., Ozorowski, G., Chuang, G.-Y., Schramm, Chaim A., Wiehe, K., et al. (2016). Maturation Pathway from Germline to Broad HIV-1 Neutralizer of a CD4Mimic Antibody. Cell 165, 449-463.

Burton, D.R., and Mascola, J.R. (2015). Antibody responses to envelope glycoproteins in HIV-1 infection. Nat Immunol 16, 571-576.

Cale, E.M., Gorman, J., Radakovich, N.A., Crooks, E.T., Osawa, K., Tong, T., Li, J., Nagarajan, R., Ozorowski, G., Ambrozak, D.R., et al. (2017). Viruslike Particles Identify an HIV V1V2 Apex-Binding Neutralizing Antibody that Lacks a Protruding Loop. Immunity 46, 777-791 e710.

Chuang, G.Y., Zhou, J., Acharya, P., Rawi, R., Shen, C.H., Sheng, Z., Zhang, B., Zhou, T., Bailer, R.T., Dandey, V.P., et al. (2019). Structural Survey of Broadly Neutralizing Antibodies Targeting the HIV-1 Env Trimer Delineates Epitope Categories and Characteristics of Recognition. Structure 27, 196206 e196.

Corey, L., Gilbert, P.B., Juraska, M., Montefiori, D.C., Morris, L., Karuna, S.T., Edupuganti, S., Mgodi, N.M., deCamp, A.C., Rudnicki, E., et al. (2021). Two Randomized Trials of Neutralizing Antibodies to Prevent HIV-1 Acquisition. N Engl J Med 384, 1003-1014.

Dai, K., He, L., Khan, S.N., O'Dell, S., McKee, K., Tran, K., Li, Y., Sundling, C., 
Morris, C.D., Mascola, J.R., et al. (2015). Rhesus Macaque B-Cell Responses to an HIV-1 Trimer Vaccine Revealed by Unbiased Longitudinal Repertoire Analysis. mBio 6, e01375-01315.

Davis, I.W., Murray, L.W., Richardson, J.S., and Richardson, D.C. (2004). MOLPROBITY: structure validation and all-atom contact analysis for nucleic acids and their complexes. Nucleic Acids Res 32, W615-619.

Doria-Rose, N., Bailer, R., Louder, M., Lin, C., Turk, E., Laub, L., Longo, N., Connors, M., and Mascola, J. (2013). High throughput HIV-1 microneutralization assay. Protocol Exchange.

Doria-Rose, N.A., Schramm, C.A., Gorman, J., Moore, P.L., Bhiman, J.N., DeKosky, B.J., Ernandes, M.J., Georgiev, I.S., Kim, H.J., Pancera, M., et al. (2014). Developmental pathway for potent V1V2-directed HIVneutralizing antibodies. Nature 509, 55-62.

Dubrovskaya, V., Tran, K., Ozorowski, G., Guenaga, J., Wilson, R., Bale, S., Cottrell, C.A., Turner, H.L., Seabright, G., O'Dell, S., et al. (2019). Vaccination with Glycan-Modified HIV NFL Envelope Trimer-Liposomes Elicits Broadly Neutralizing Antibodies to Multiple Sites of Vulnerability. Immunity 51, 915-929 e917.

Dunbar, J., Krawczyk, K., Leem, J., Marks, C., Nowak, J., Regep, C., Georges, G., Kelm, S., Popovic, B., and Deane, C.M. (2016). SAbPred: a structurebased antibody prediction server. Nucleic Acids Res 44, W474-478.

Emsley, P., and Cowtan, K. (2004). Coot: model-building tools for molecular graphics. Acta Crystallogr D Biol Crystallogr 60, 2126-2132.

Fera, D., Schmidt, A.G., Haynes, B.F., Gao, F., Liao, H.X., Kepler, T.B., and Harrison, S.C. (2014). Affinity maturation in an HIV broadly neutralizing Bcell lineage through reorientation of variable domains. Proc Natl Acad Sci U S A 111, 10275-10280.

Gao, F., Bonsignori, M., Liao, H.X., Kumar, A., Xia, S.M., Lu, X., Cai, F., Hwang, K.K., Song, H., Zhou, T., et al. (2014). Cooperation of B Cell Lineages in Induction of HIV-1-Broadly Neutralizing Antibodies. Cell 158, 481-491. 
Gao, N., Gai, Y., Meng, L., Wang, C., Zhang, X., Wang, W., Qin, C., Yu, X., and Gao, F. (2020). Development of Antibodies with Broad Neutralization Specificities against HIV-1 after Long Term SHIV Infection in Macaques. Viruses 12.

Gao, N., Wang, W., Wang, C., Gu, T., Guo, R., Yu, B., Kong, W., Qin, C., Giorgi, E.E., Chen, Z., et al. (2018). Development of broad neutralization activity in simian/human immunodeficiency virus-infected rhesus macaques after long-term infection. AIDS 32, 555-563.

Gautam, R., Nishimura, Y., Pegu, A., Nason, M.C., Klein, F., Gazumyan, A., Golijanin, J., Buckler-White, A., Sadjadpour, R., Wang, K., et al. (2016). A single injection of anti-HIV-1 antibodies protects against repeated SHIV challenges. Nature 533, 105-109.

Gorman, J., Chuang, G.Y., Lai, Y.T., Shen, C.H., Boyington, J.C., Druz, A., Geng, H., Louder, M.K., McKee, K., Rawi, R., et al. (2020). Structure of SuperPotent Antibody CAP256-VRC26.25 in Complex with HIV-1 Envelope Reveals a Combined Mode of Trimer-Apex Recognition. Cell Rep 31, 107488.

Gorman, J., Soto, C., Yang, M.M., Davenport, T.M., Guttman, M., Bailer, R.T., Chambers, M., Chuang, G.Y., DeKosky, B.J., Doria-Rose, N.A., et al. (2016). Structures of HIV-1 Env V1V2 with broadly neutralizing antibodies reveal commonalities that enable vaccine design. Nat Struct Mol Biol 23, 81-90.

Gouy, M., Guindon, S., and Gascuel, O. (2010). SeaView version 4: A multiplatform graphical user interface for sequence alignment and phylogenetic tree building. Mol Biol Evol 27, 221-224.

Gray, G.E., Bekker, L.G., Laher, F., Malahleha, M., Allen, M., Moodie, Z., Grunenberg, N., Huang, Y., Grove, D., Prigmore, B., et al. (2021). Vaccine Efficacy of ALVAC-HIV and Bivalent Subtype C gp120-MF59 in Adults. N Engl J Med 384, 1089-1100.

Han, Q., Jones, J.A., Nicely, N.I., Reed, R.K., Shen, X., Mansouri, K., Louder, 
M., Trama, A.M., Alam, S.M., Edwards, R.J., et al. (2019). Difficult-toneutralize global HIV-1 isolates are neutralized by antibodies targeting open envelope conformations. Nat Commun 10, 2898.

Havenar-Daughton, C., Carnathan, D.G., Torrents de la Pena, A., Pauthner, M., Briney, B., Reiss, S.M., Wood, J.S., Kaushik, K., van Gils, M.J., Rosales, S.L., et al. (2016). Direct Probing of Germinal Center Responses Reveals Immunological Features and Bottlenecks for Neutralizing Antibody Responses to HIV Env Trimer. Cell Rep 17, 2195-2209.

Haynes, B.F., Burton, D.R., and Mascola, J.R. (2019). Multiple roles for HIV broadly neutralizing antibodies. Sci Transl Med 11.

Hessell, A.J., and Haigwood, N.L. (2015). Animal models in HIV-1 protection and therapy. Curr Opin HIV AIDS 10, 170-176.

Hraber, P., Seaman, M.S., Bailer, R.T., Mascola, J.R., Montefiori, D.C., and Korber, B.T. (2014). Prevalence of broadly neutralizing antibody responses during chronic HIV-1 infection. AIDS 28, 163-169.

Kepler, T.B. (2013). Reconstructing a B-cell clonal lineage. I. Statistical inference of unobserved ancestors. F1000Res 2, 103.

Klein, F., Mouquet, H., Dosenovic, P., Scheid, J.F., Scharf, L., and Nussenzweig, M.C. (2013). Antibodies in HIV-1 vaccine development and therapy. Science 341, 1199-1204.

Kong, L., He, L., de Val, N., Vora, N., Morris, C.D., Azadnia, P., Sok, D., Zhou, B., Burton, D.R., Ward, A.B., et al. (2016). Uncleaved prefusion-optimized gp140 trimers derived from analysis of HIV-1 envelope metastability. Nat Commun 7, 12040.

Kong, R., Duan, H., Sheng, Z., Xu, K., Acharya, P., Chen, X., Cheng, C., Dingens, A.S., Gorman, J., Sastry, M., et al. (2019). Antibody Lineages with Vaccine-Induced Antigen-Binding Hotspots Develop Broad HIV Neutralization. Cell 178, 567-584 e519.

Krissinel, E., and Henrick, K. (2007). Inference of macromolecular assemblies from crystalline state. J Mol Biol 372, 774-797. 
Kwong, P.D., and Mascola, J.R. (2018). HIV-1 Vaccines Based on Antibody Identification, B Cell Ontogeny, and Epitope Structure. Immunity 48, 855871.

Kwong, P.D., Mascola, J.R., and Nabel, G.J. (2013). Broadly neutralizing antibodies and the search for an HIV-1 vaccine: the end of the beginning. Nat Rev Immunol 13, 693-701.

Landais, E., Huang, X., Havenar-Daughton, C., Murrell, B., Price, M.A., Wickramasinghe, L., Ramos, A., Bian, C.B., Simek, M., Allen, S., et al. (2016). Broadly Neutralizing Antibody Responses in a Large Longitudinal Sub-Saharan HIV Primary Infection Cohort. PLoS Pathog 12, e1005369. Larkin, M.A., Blackshields, G., Brown, N.P., Chenna, R., McGettigan, P.A., McWilliam, H., Valentin, F., Wallace, I.M., Wilm, A., Lopez, R., et al. (2007). Clustal W and Clustal X version 2.0. Bioinformatics 23, 2947-2948.

Lee, J.H., Andrabi, R., Su, C.Y., Yasmeen, A., Julien, J.P., Kong, L., Wu, N.C., McBride, R., Sok, D., Pauthner, M., et al. (2017). A Broadly Neutralizing Antibody Targets the Dynamic HIV Envelope Trimer Apex via a Long, Rigidified, and Anionic beta-Hairpin Structure. Immunity 46, 690-702.

Liao, H.X., Levesque, M.C., Nagel, A., Dixon, A., Zhang, R., Walter, E., Parks, R., Whitesides, J., Marshall, D.J., Hwang, K.K., et al. (2009). Highthroughput isolation of immunoglobulin genes from single human B cells and expression as monoclonal antibodies. J Virol Methods 158, 171-179. Liao, H.X., Lynch, R., Zhou, T., Gao, F., Alam, S.M., Boyd, S.D., Fire, A.Z., Roskin, K.M., Schramm, C.A., Zhang, Z., et al. (2013). Co-evolution of a broadly neutralizing HIV-1 antibody and founder virus. Nature 496, 469-476. Malim, M.H., and Emerman, M. (2001). HIV-1 sequence variation: drift, shift, and attenuation. Cell 104, 469-472.

McCoy, A.J. (2007). Solving structures of protein complexes by molecular replacement with Phaser. Acta Crystallogr D Biol Crystallogr 63, 32-41.

McLellan, J.S., Pancera, M., Carrico, C., Gorman, J., Julien, J.P., Khayat, R., Louder, R., Pejchal, R., Sastry, M., Dai, K., et al. (2011). Structure of HIV-1 
gp120 V1/V2 domain with broadly neutralizing antibody PG9. Nature 480, 336-343.

Moore, P.L., Gorman, J., Doria-Rose, N.A., and Morris, L. (2017). Ontogenybased immunogens for the induction of V2-directed HIV broadly neutralizing antibodies. Immunol Rev 275, 217-229.

Moore, P.L., Gray, E.S., Sheward, D., Madiga, M., Ranchobe, N., Lai, Z., Honnen, W.J., Nonyane, M., Tumba, N., Hermanus, T., et al. (2011). Potent and broad neutralization of HIV-1 subtype $\mathrm{C}$ by plasma antibodies targeting a quaternary epitope including residues in the V2 loop. J Virol 85, 31283141.

Pauthner, M.G., Nkolola, J.P., Havenar-Daughton, C., Murrell, B., Reiss, S.M., Bastidas, R., Prevost, J., Nedellec, R., von Bredow, B., Abbink, P., et al. (2019). Vaccine-Induced Protection from Homologous Tier 2 SHIV Challenge in Nonhuman Primates Depends on Serum-Neutralizing Antibody Titers. Immunity 50, 241-252 e246.

Pegu, A., Hessell, A.J., Mascola, J.R., and Haigwood, N.L. (2017). Use of broadly neutralizing antibodies for HIV-1 prevention. Immunol Rev 275, 296-312.

Pettersen, E.F., Goddard, T.D., Huang, C.C., Couch, G.S., Greenblatt, D.M., Meng, E.C., and Ferrin, T.E. (2004). UCSF Chimera--a visualization system for exploratory research and analysis. J Comput Chem 25, 1605-1612.

Pettersen, E.F., Goddard, T.D., Huang, C.C., Meng, E.C., Couch, G.S., Croll, T.I., Morris, J.H., and Ferrin, T.E. (2021). UCSF ChimeraX: Structure visualization for researchers, educators, and developers. Protein Sci 30, 70-82.

Piehler, B., Nelson, E.K., Eckels, J., Ramsay, S., Lum, K., Wood, B., Greene, K.M., Gao, H., Seaman, M.S., Montefiori, D.C., et al. (2011). LabKey Server NAb: a tool for analyzing, visualizing and sharing results from neutralizing antibody assays. BMC Immunol 12, 33.

Punjani, A., Rubinstein, J.L., Fleet, D.J., and Brubaker, M.A. (2017). 
cryoSPARC: algorithms for rapid unsupervised cryo-EM structure determination. Nat Methods 14, 290-296.

Rahman, M.A., and Robert-Guroff, M. (2019). Accelerating HIV vaccine development using non-human primate models. Expert Rev Vaccines 18, 61-73.

Rawi, R., Rutten, L., Lai, Y.T., Olia, A.S., Blokland, S., Juraszek, J., Shen, C.H., Tsybovsky, Y., Verardi, R., Yang, Y., et al. (2020). Automated Design by Structure-Based Stabilization and Consensus Repair to Achieve PrefusionClosed Envelope Trimers in a Wide Variety of HIV Strains. Cell Rep 33, 108432.

Rerks-Ngarm, S., Pitisuttithum, P., Nitayaphan, S., Kaewkungwal, J., Chiu, J., Paris, R., Premsri, N., Namwat, C., de Souza, M., Adams, E., et al. (2009). Vaccination with ALVAC and AIDSVAX to prevent HIV-1 infection in Thailand. N Engl J Med 361, 2209-2220.

Roark, R.S., Li, H., Williams, W.B., Chug, H., Mason, R.D., Gorman, J., Wang, S., Lee, F.H., Rando, J., Bonsignori, M., et al. (2021). Recapitulation of HIV1 Env-antibody coevolution in macaques leading to neutralization breadth. Science 371.

Sanders, R.W., van Gils, M.J., Derking, R., Sok, D., Ketas, T.J., Burger, J.A., Ozorowski, G., Cupo, A., Simonich, C., Goo, L., et al. (2015). HIV-1 VACCINES. HIV-1 neutralizing antibodies induced by native-like envelope trimers. Science 349, aac4223.

Sarzotti-Kelsoe, M., Bailer, R.T., Turk, E., Lin, C.L., Bilska, M., Greene, K.M., Gao, H., Todd, C.A., Ozaki, D.A., Seaman, M.S., et al. (2014). Optimization and validation of the TZM-bl assay for standardized assessments of neutralizing antibodies against HIV-1. J Immunol Methods 409, 131-146.

Sellhorn, G., Caldwell, Z., Mineart, C., and Stamatatos, L. (2009). Improving the expression of recombinant soluble HIV Envelope glycoproteins using pseudo-stable transient transfection. Vaccine $28,430-436$.

Shen, C.H., DeKosky, B.J., Guo, Y., Xu, K., Gu, Y., Kilam, D., Ko, S.H., Kong, 
R., Liu, K., Louder, M.K., et al. (2020). VRC34-Antibody Lineage Development Reveals How a Required Rare Mutation Shapes the Maturation of a Broad HIV-Neutralizing Lineage. Cell Host Microbe 27, 531543 e536.

Siddappa, N.B., Watkins, J.D., Wassermann, K.J., Song, R., Wang, W., Kramer, V.G., Lakhashe, S., Santosuosso, M., Poznansky, M.C., Novembre, F.J., et al. (2010). R5 clade C SHIV strains with tier 1 or 2 neutralization sensitivity: tools to dissect env evolution and to develop AIDS vaccines in primate models. PLoS One 5, e11689.

Sok, D., and Burton, D.R. (2018). Recent progress in broadly neutralizing antibodies to HIV. Nat Immunol 19, 1179-1188.

Suloway, C., Pulokas, J., Fellmann, D., Cheng, A., Guerra, F., Quispe, J., Stagg, S., Potter, C.S., and Carragher, B. (2005). Automated molecular microscopy: the new Leginon system. J Struct Biol 151, 41-60.

Sundling, C., Li, Y., Huynh, N., Poulsen, C., Wilson, R., O'Dell, S., Feng, Y., Mascola, J.R., Wyatt, R.T., and Karlsson Hedestam, G.B. (2012a). Highresolution definition of vaccine-elicited $B$ cell responses against the HIV primary receptor binding site. Sci Transl Med 4, 142ra196.

Sundling, C., Phad, G., Douagi, I., Navis, M., and Karlsson Hedestam, G.B. (2012b). Isolation of antibody V(D)J sequences from single cell sorted rhesus macaque B cells. J Immunol Methods 386, 85-93.

UNAIDS (2021). UNAIDS Fact-sheet 2020. [Online]. Available: https://www.unaids.org/en/resources/fact-sheet [Accessed].

Walker, L.M., Phogat, S.K., Chan-Hui, P.Y., Wagner, D., Phung, P., Goss, J.L., Wrin, T., Simek, M.D., Fling, S., Mitcham, J.L., et al. (2009). Broad and potent neutralizing antibodies from an African donor reveal a new HIV-1 vaccine target. Science 326, 285-289.

Wang, Y., O'Dell, S., Turner, H.L., Chiang, C.I., Lei, L., Guenaga, J., Wilson, R., Martinez-Murillo, P., Doria-Rose, N., Ward, A.B., et al. (2017). HIV-1 CrossReactive Primary Virus Neutralizing Antibody Response Elicited by 
Immunization in Nonhuman Primates. J Virol 91.

Wang, Z., Barnes, C.O., Gautam, R., Cetrulo Lorenzi, J.C., Mayer, C.T., Oliveira, T.Y., Ramos, V., Cipolla, M., Gordon, K.M., Gristick, H.B., et al. (2020). A broadly neutralizing macaque monoclonal antibody against the HIV-1 V3Glycan patch. Elife 9.

Wiehe, K., Easterhoff, D., Luo, K., Nicely, N.I., Bradley, T., Jaeger, F.H., Dennison, S.M., Zhang, R., Lloyd, K.E., Stolarchuk, C., et al. (2014). Antibody light-chain-restricted recognition of the site of immune pressure in the RV144 HIV-1 vaccine trial is phylogenetically conserved. Immunity 41, 909-918.

Williams, W.B., Zhang, J., Jiang, C., Nicely, N.I., Fera, D., Luo, K., Moody, M.A., Liao, H.X., Alam, S.M., Kepler, T.B., et al. (2017). Initiation of HIV neutralizing B cell lineages with sequential envelope immunizations. Nat Commun 8, 1732.

Xu, K., Acharya, P., Kong, R., Cheng, C., Chuang, G.Y., Liu, K., Louder, M.K., O'Dell, S., Rawi, R., Sastry, M., et al. (2018). Epitope-based vaccine design yields fusion peptide-directed antibodies that neutralize diverse strains of HIV-1. Nat Med 24, 857-867.

Zhang, R., Verkoczy, L., Wiehe, K., Munir Alam, S., Nicely, N.I., Santra, S., Bradley, T., Pemble, C.W.t., Zhang, J., Gao, F., et al. (2016). Initiation of immune tolerance-controlled HIV gp41 neutralizing B cell lineages. Sci Transl Med 8, 336ra362. 
A Neutralizing titers $\left(\mathrm{ID}_{50}\right)$ of plasma from $\mathrm{G} 1015 \mathrm{R}$

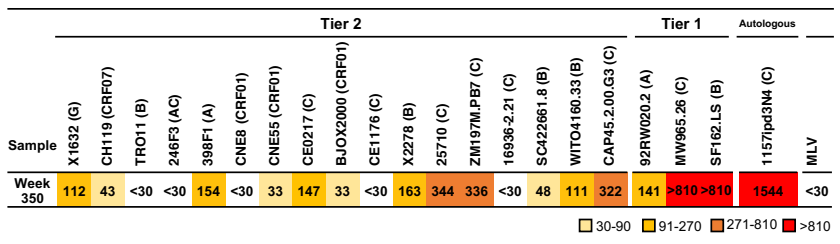

B V1V2-specific B cell sorting

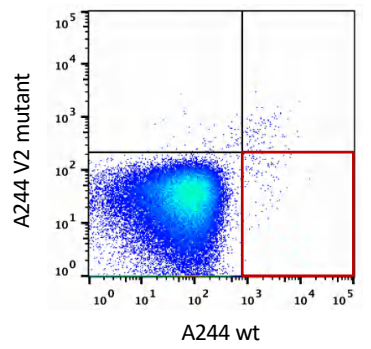

C Neutralization titers $\left(\mathrm{IC}_{50}\right)$ of mAbs isolated from $\mathrm{G} 1015 \mathrm{R}$

\begin{tabular}{|c|c|c|c|c|c|c|c|c|c|c|c|c|c|c|c|c|c|c|c|c|c|c|}
\hline \multirow[b]{2}{*}{ mAb ID } & \multicolumn{17}{|c|}{ Tier 2} & \multicolumn{3}{|c|}{ Tier 1} & \multicolumn{2}{|l|}{ Autologous } \\
\hline & $\begin{array}{l}\overline{0} \\
\text { N } \\
\tilde{O} \\
\bar{x}\end{array}$ & 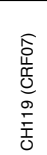 & $\begin{array}{l}\widehat{\underline{M}} \\
\bar{\sigma} \\
\underline{\underline{\alpha}}\end{array}$ & 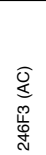 & 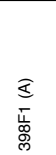 & 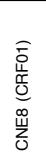 & 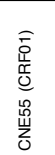 & $\begin{array}{l}\widehat{0} \\
\hat{N} \\
\stackrel{\tilde{U}}{u}\end{array}$ & 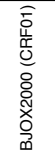 & $\begin{array}{l}\widehat{O} \\
\stackrel{\rho}{\circ} \\
\stackrel{\bar{U}}{0}\end{array}$ & $\begin{array}{l}\widehat{\underline{m}} \\
\infty \\
\hat{N} \\
\tilde{x}\end{array}$ & \begin{tabular}{l}
$\widehat{0}$ \\
0 \\
\multirow{0}{*}{} \\
N
\end{tabular} & 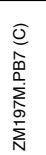 & 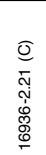 & 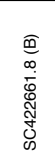 &  & 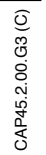 & 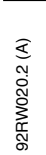 & 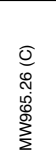 & 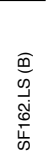 & 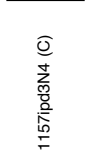 & $\vec{\Sigma}$ \\
\hline J038 & 41.67 & 29.90 & 13.80 & 25.42 & 29.41 & $>50$ & 20.83 & 5.54 & 26.32 & $>50$ & 1.28 & 0.87 & $>50$ & $>50$ & 37.50 & 0.76 & $\overline{0.48}$ & $>50$ & 0.11 & $>50$ & 0.02 & $>50$ \\
\hline J033 & $>50$ & 18.05 & 20.59 & 21.43 & 38.46 & $>50$ & 15.00 & 5.14 & 14.85 & $>50$ & 1.78 & 1.56 & $>50$ & $>50$ & 20.83 & 0.19 & 0.23 & $>50$ & 0.31 & $>50$ & 0.03 & $>50$ \\
\hline J029 & $>50$ & $>50$ & 43.48 & $>50$ & $>50$ & $>50$ & 29.41 & 4.44 & $>50$ & $>50$ & 1.25 & 0.81 & $>50$ & $>50$ & 4.73 & 0.28 & 0.14 & $>50$ & 0.10 & $>50$ & 0.08 & $>50$ \\
\hline J040 & $>50$ & $>50$ & $>50$ & $>50$ & $>50$ & $>50$ & 48.29 & 4.35 & $>50$ & $>50$ & 2.27 & 1.72 & $>50$ & $>50$ & $>50$ & 0.61 & 0.25 & $>50$ & 0.04 & $>50$ & 0.09 & $>50$ \\
\hline J031 & $>50$ & $>50$ & 37.03 & $>50$ & $>50$ & $>50$ & 47.61 & $>50$ & $>50$ & $>50$ & 0.78 & 15.42 & $>50$ & $>50$ & 9.71 & 6.67 & 2.99 & $>50$ & 0.06 & $>50$ & 0.16 & $>50$ \\
\hline J044 & $>50$ & $>50$ & $>50$ & $>50$ & $>50$ & $>50$ & 44.12 & $>50$ & $>50$ & $>50$ & 3.75 & 9.47 & $>50$ & $>50$ & $>50$ & 27.78 & $>50$ & $>50$ & 0.96 & $>50$ & 0.25 & $>50$ \\
\hline J039 & $>50$ & $>50$ & $>50$ & $>50$ & $>50$ & $>50$ & $>50$ & $>50$ & $>50$ & $>50$ & $>50$ & $>50$ & $>50$ & $>50$ & $>50$ & $>50$ & $>50$ & $>50$ & 0.21 & $>50$ & 4.97 & $>50$ \\
\hline J024 & $>50$ & $>50$ & $>50$ & $>50$ & $>50$ & $>50$ & $>50$ & $>50$ & $>50$ & $>50$ & $>50$ & $>50$ & $>50$ & $>50$ & $>20$ & $>50$ & $>50$ & $>20$ & 0.4 & 0.64 & 0.62 & $>50$ \\
\hline J013 & $>50$ & $>50$ & $>50$ & $>50$ & $>50$ & $>50$ & $>50$ & $>50$ & $>50$ & $>50$ & $>50$ & $>50$ & $>50$ & $>50$ & $>50$ & $>50$ & $>50$ & $>50$ & $>50$ & $>50$ & 8.16 & $>50$ \\
\hline J037 & $>50$ & $>50$ & $>50$ & $>50$ & $>50$ & $>50$ & $>50$ & $>50$ & $>50$ & $>50$ & $>50$ & $>50$ & $>50$ & $>50$ & $>50$ & $>50$ & $>50$ & $>50$ & 0.20 & 0.73 & 0.39 & $>50$ \\
\hline J032 & $>50$ & $>50$ & $>50$ & $>50$ & $>50$ & $>50$ & $>50$ & $>50$ & $>50$ & $>50$ & $>50$ & 27 & $>50$ & $>50$ & $>50$ & $>50$ & $>50$ & $>50$ & 0.01 & 0.17 & 0.96 & $>50$ \\
\hline J007 & $>50$ & $>50$ & $>50$ & $>50$ & $>50$ & $>50$ & $>50$ & $>50$ & $>50$ & $>50$ & $>50$ & $>50$ & $>50$ & $>50$ & $>50$ & $>50$ & $>50$ & $>50$ & 1.25 & $>50$ & 0.98 & $>50$ \\
\hline
\end{tabular}

D J038 broadly neutralizes HIV-1 and clusters with V1V2-targeting antibodies
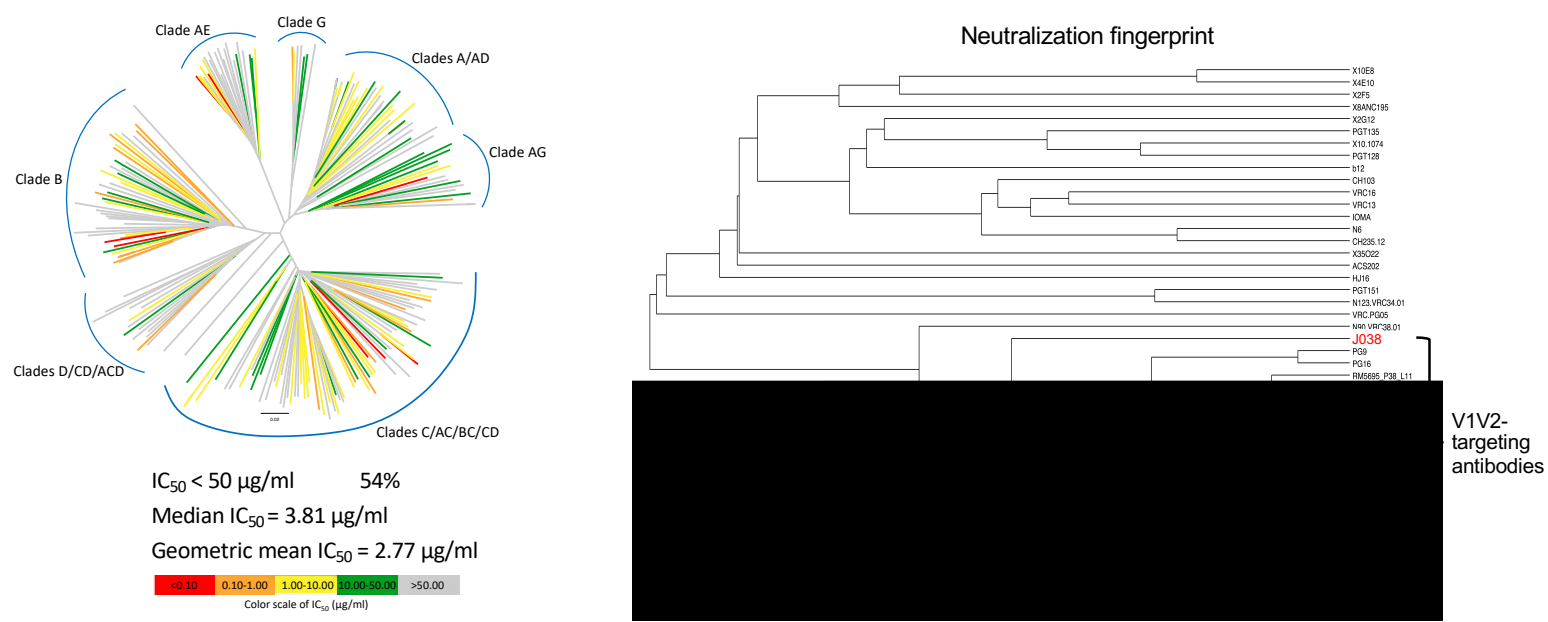

Color scale of $\mathrm{I} \mathrm{C}_{50}(\mathrm{\mu g} / \mathrm{ml})$

Figure 1. Isolation of broadly neutralizing antibodies from G1015R

(A) Neutralization breadth of week 350 plasma post infection from G1015R infected with $\mathrm{SHIV}_{1157 \mathrm{ipd} 3 \mathrm{~N} 4}$. Data are shown as $\mathrm{ID}_{50}$ and neutralization potency is color coded.

(B) Fluorescence-activated cell sorting (FACS) plots of sorted V1V2-specific single B cells from week 350 peripheral blood mononuclear cells (PBMCs) from G1015R that bind d11_A244_gp120_SavBV421 but not glycan knockout mutant N156_160K_d11_A244 gp120_SavAF647 (red box).

(C) Neutralization profiles of $12 \mathrm{mAbs}$ were determined with 17 tier 2 viruses, three tier 1 viruses and the autologous virus. Murine leukemia virus (MLV) was used as a nonspecific control. Neutralization titers are represented as $\mathrm{IC}_{50}(\mu \mathrm{g} / \mathrm{mL})$.

(D) J038 neutralizes 112 strains of a 208-virus panel (54\%). Stains in HIV-1 phylogenetic tree are colored according to their respective $\mathrm{J} 038$ potency based on $\mathrm{IC}_{50}$. Median and geometric mean titers are calculated only for samples with $\mathrm{IC}_{50}<50 \mu \mathrm{g} / \mathrm{ml}$. Neutralization profile of J038 shows that it clusters with V1V2-targeting antibodies.

See also Figures S1-S2 and Table S1-S2. 
A Cryo-EM structure of the J038-Env complex at $3.4 \AA$ resolution
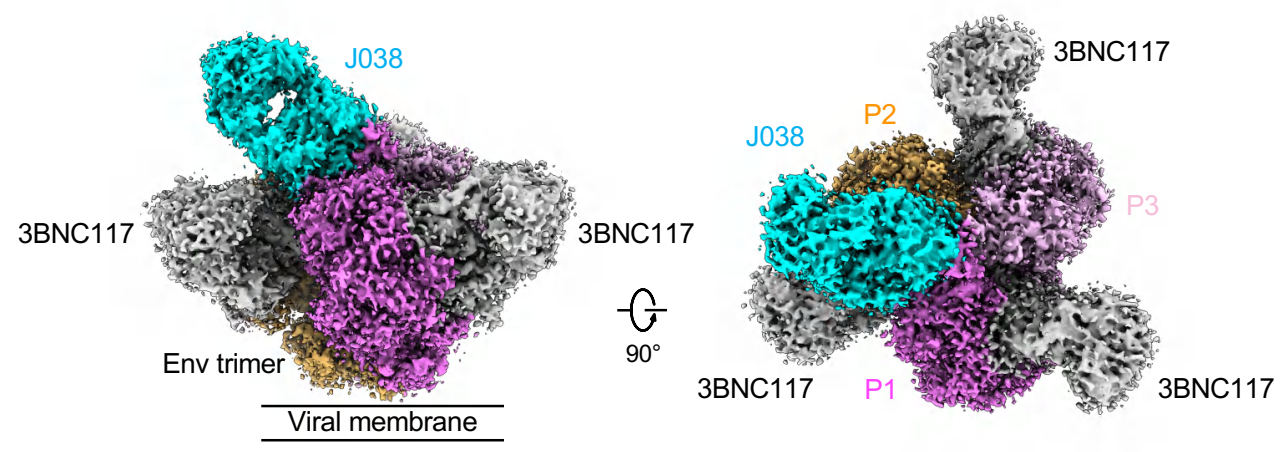

B

J038 in complex with HIV-1 C1080 Env

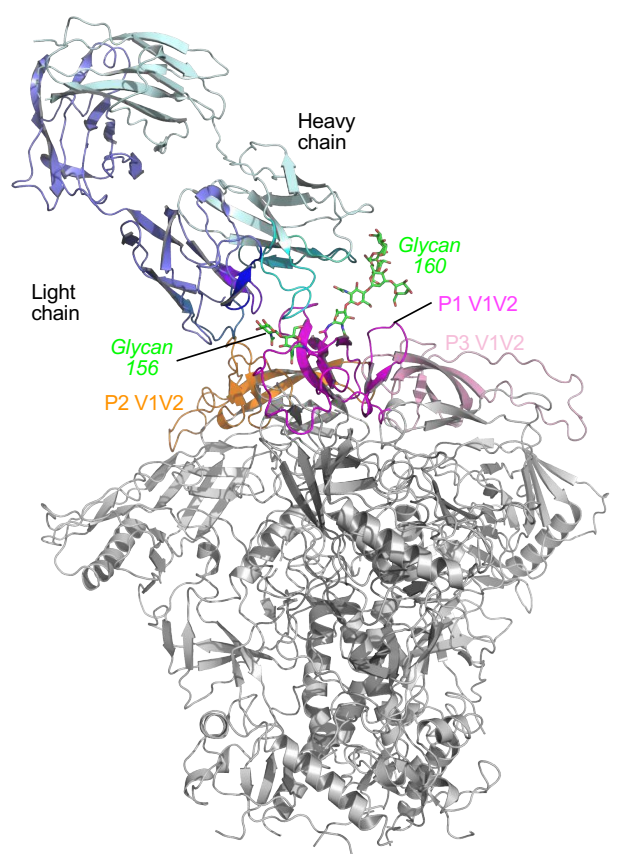

C J038 interaction with V2 loops of neighboring protomers

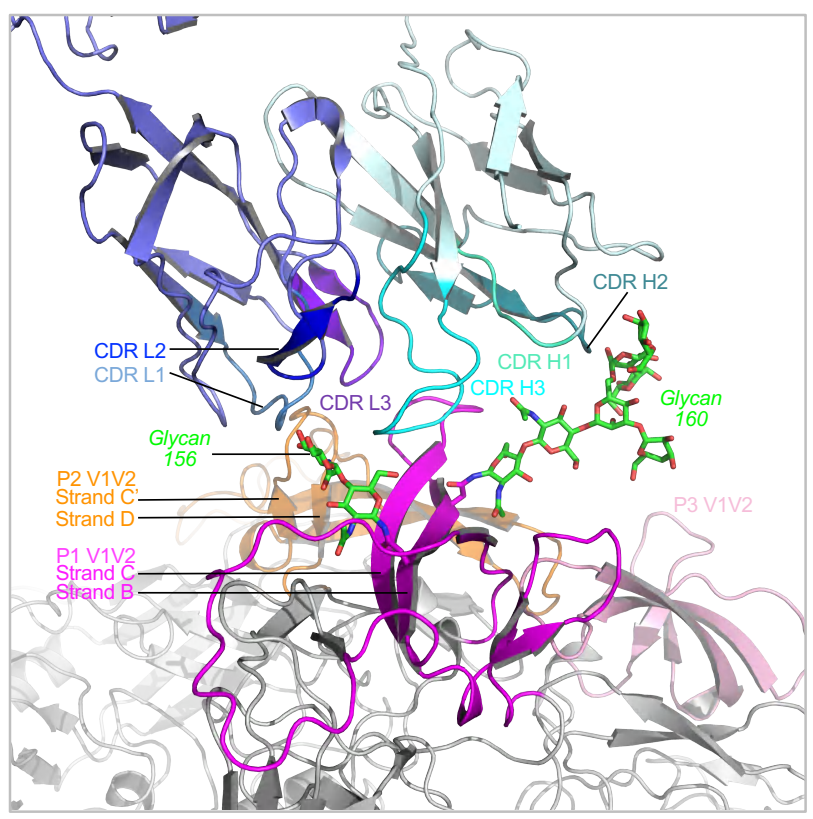

Figure 2. Antibody J038 targets the V2-apex of the HIV-1 Env.

(A) Overall cryo-EM structure of the J038 Fab -Env complex, with EM reconstruction density shown in gray. The CD4-binding site antibody 3BNC117 was used to aid the resolution. Protomers of the trimer are labeled as P1, P2 and P3.

(B) Antibody J038 recognition of the HIV-1 Env. Heavy and light chains of J038 are colored in cyan and blue, respectively. The V1V2 loops of the Env are colored magenta, pink and orange for the three protomers, respectively. Glycans N156 and N160 on P1 interacting with J038 are colored green. The CD4-binding site antibody 3BNC117 was omitted for clarify.

(C) J038 interaction with V2-loops of the neighboring protomers P1 and P2. The complementarity determining regions (CDR) of J038 are shown in shades of cyan and blue. The strands of V2 that interacted with J038 are labeled.

See also Figures S5 and Tables S1-S4. 
A Interactions between $\mathrm{J} 038$ and $\mathrm{V} 2$ of protomers $\mathrm{P} 1$ and $\mathrm{P} 2$
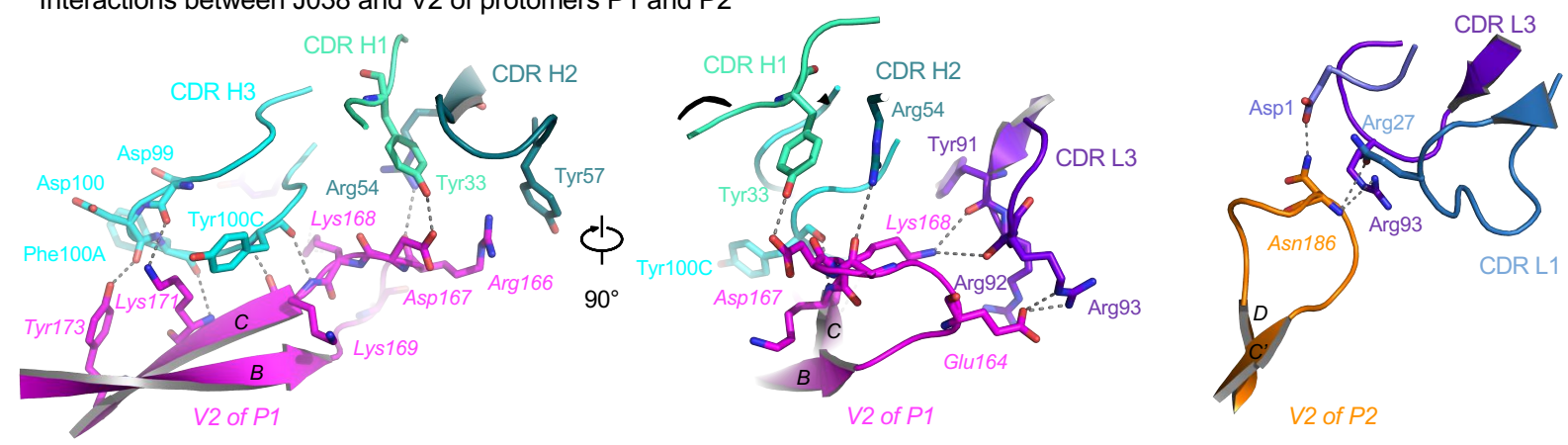

B J038 interactions with Glycans on N156 and N160
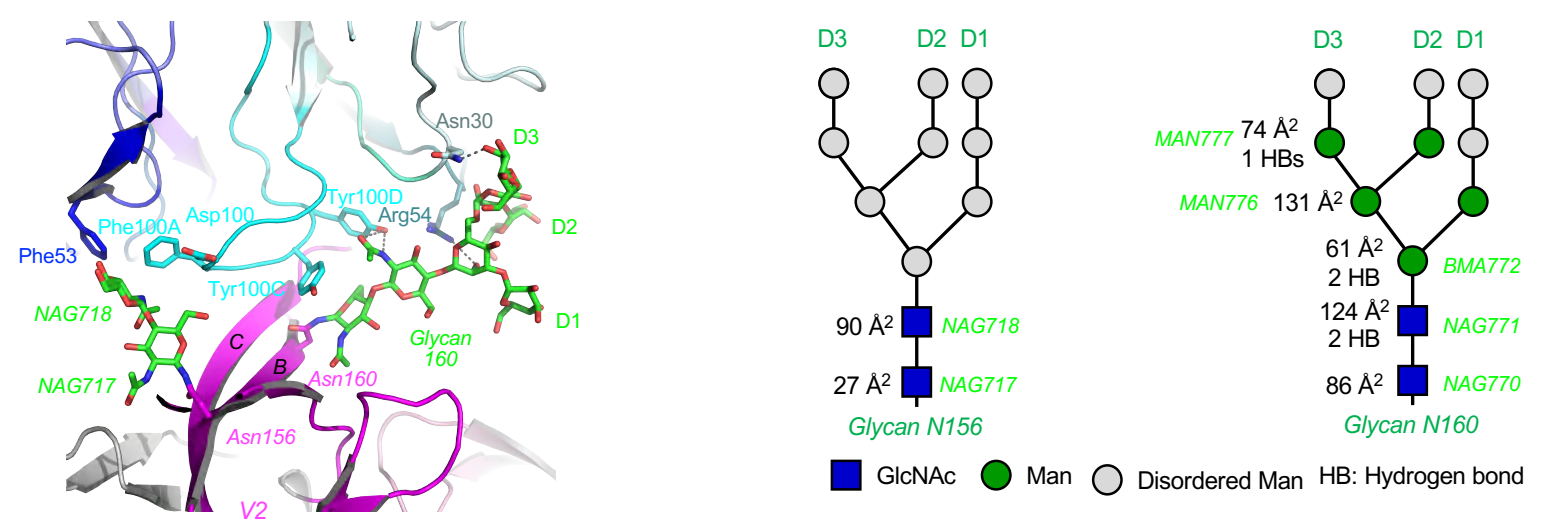

GlcNAc $\bigcirc$ Man $\bigcirc$ Disordered Man HB: Hydrogen bond

Figure 3. J038 interaction with V2-apex of HIV-1 Env.

(A) J038 interaction of V2 of protomers P1 (left and middle) and P2 (right). Key interacting residues are shown in sticks and numbered with hydrogen bonds shown in dashed lines.

(B) Interactions between J038 and glycans on Asn156 and Asn160. Key residues that interact with glycans are shown in sticks and numbered with hydrogen bonds shown in dashed lines. A schematic for each glycan are shown next to the cryo-EM structure with GlcNAc as blue squares and mannose residues as green circles. Buried surface areas $\left(\AA^{2}\right)$ and hydrogen bonds with J038 for each glycan moieties are labeled.

See also Table S3-4. 
A Mapping of J038 epitope residues onto the V2 sequences

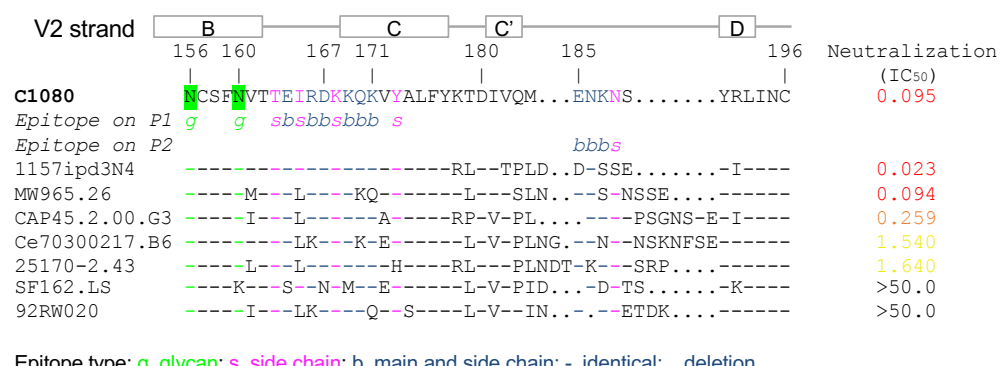

Epitope type: g, glycan; s, side chain; b, main and side chain; -, identical; ., deletion.

B Effects of mutation in V2 loop on neutralization of J038 lineage antibodies

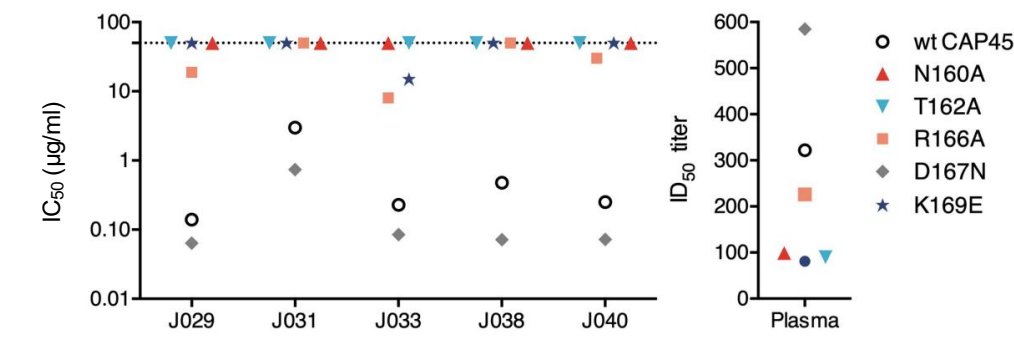

C Glycan is important for J038 binding to HIV-1 Env

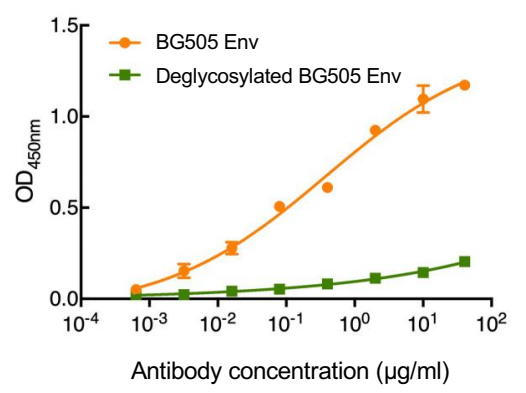

Figure 4. Key J038 residues interacting with V2-apex of HIV-1 Env.

(A) Alignment of V2 sequences from HIV-1 strains, shown relative to their neutralization sensitivities. J038 contacts are marked as $\mathrm{g}$ for glycan contacts, $\mathrm{s}$ for side chain contacts and $\mathrm{b}$ for both side chain and main chain contacts.

(B) Effects of mutations in V2 on neutralization potency of the J038 lineage antibodies. Mutations removing the Asn160 glycosylation site on V2 make the virus resistant to J038 lineage antibodies.

(C) Deglycosylation reduces binding of HIV-1 BG505 Env to J038.

See also Figure S5 and Table S4. 
A Mode of recognition by different V2-apex antibodies

J038

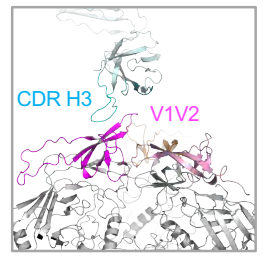

J033

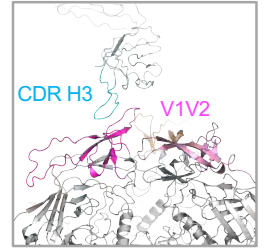

PG9

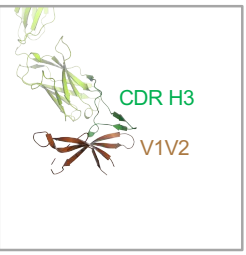

VRC38

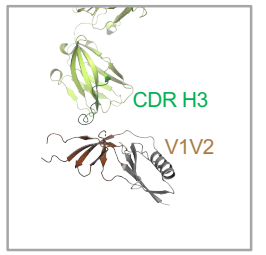

VRC26.25

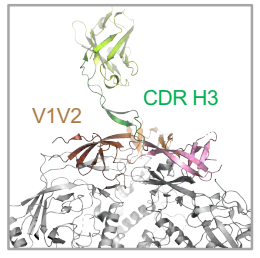

PGT145

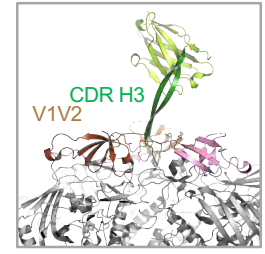

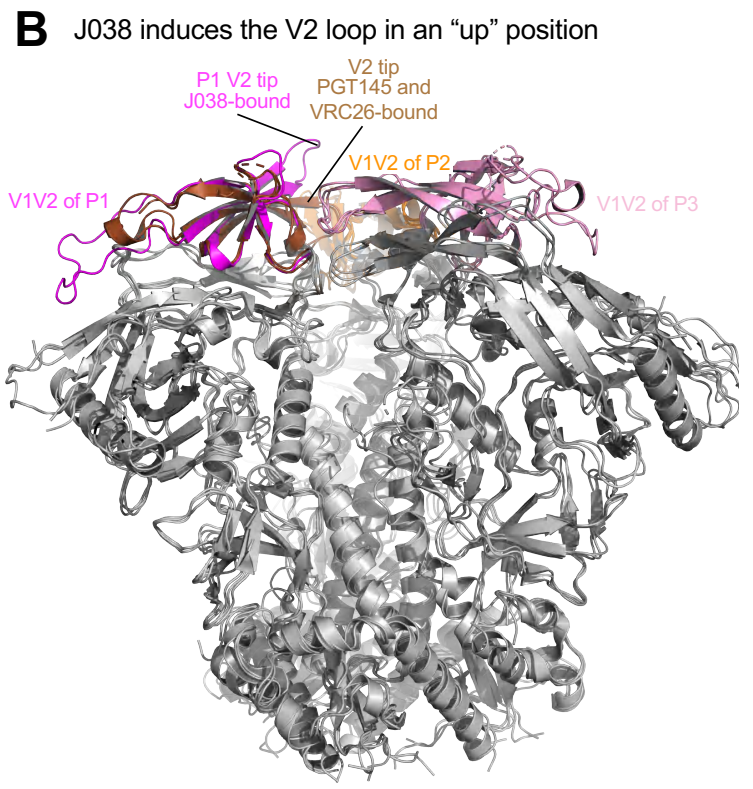

J038, PGT145 and VRC26-bound Env

C Comparison of $\mathrm{V} 2$ loop conformation


D Conformational difference between antibody-bound V2 loops in protomer P1

\begin{tabular}{ccccc}
\hline RMSD $(\AA)$ & PG9 & VRC38 & PGT145 & VRC26 \\
\hline J038 & 6.9 & 7.0 & 7.5 & 7.3 \\
PG9 & & 3.0 & 3.2 & 3.1 \\
VRC38 & & & 3.4 & 3.4 \\
PGT145 & & & & 1.7 \\
\hline
\end{tabular}

Figure 5. J038 uses a different mode of gp120 recognition.

(A) Comparison of heavy chain CDR H3 interactions with the V2-apex of HIV-1 Env. CDR H3s of antibodies (J008, J033, PG9, VRC38, VRC26 and PGT145) are shown in cyan or dark green. The V1V2 loops are colored as in Figure 1 with that of protomer 1 in the PG9-, VRC38-, VRC26- and PGT145-bound shown in brown for comparison.

(B) Binding of J038 induces the strands B and C in P1 V2-loop in an "up" conformation. The structures of J038, VRC26 and PGT145 in complex with HIV-1 Env are superposed and the V1V2 loops are colored the same as in (A).

(C) Comparison of conformation of the P1 V2-loops in J038, PG9-, VRC38-, VRC26- and PGT145-bound structures, distance shown are between $\mathrm{C} \alpha$ atoms of Glu165 at the tip of loop between strands B and C.

(D) $\mathrm{C} \alpha$-rmsd between antibody-bound V2 loop strands B and C (residues 154-177) in P1. 

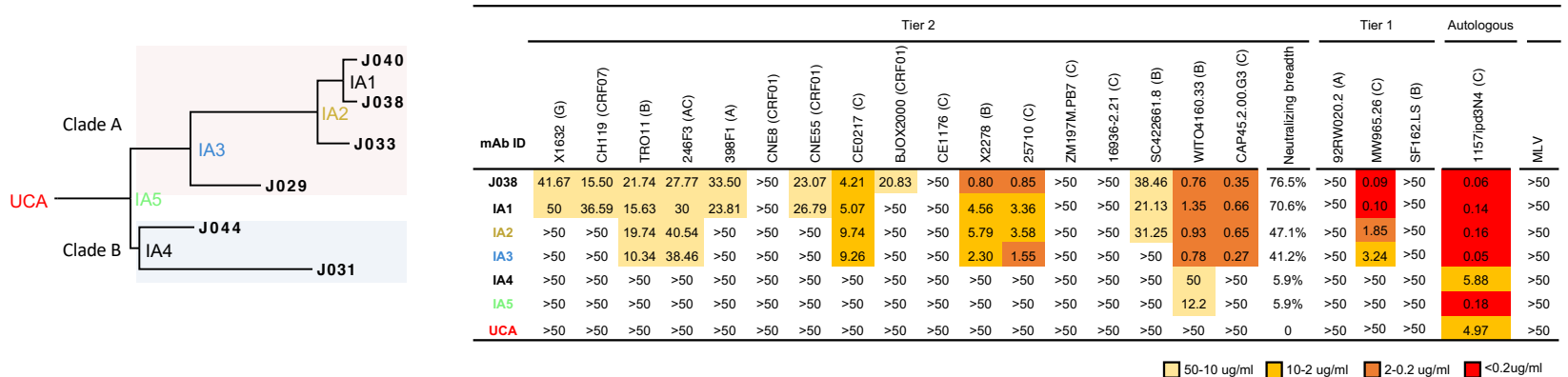

C Sequence alignment and mapping of paratope

Heavy chain

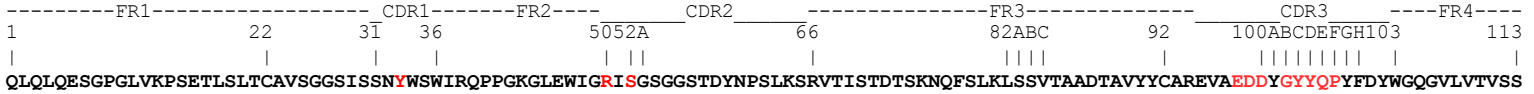

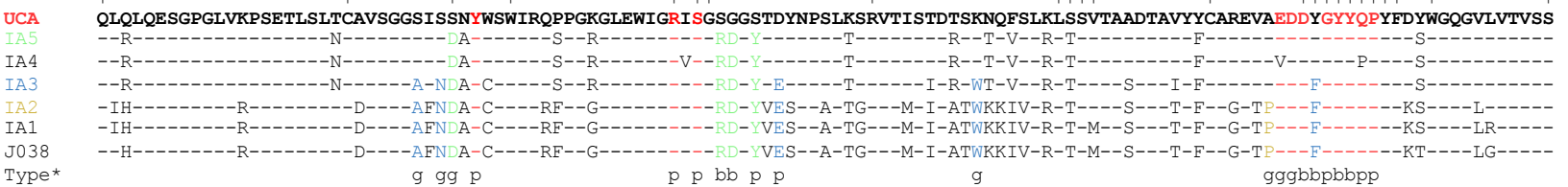

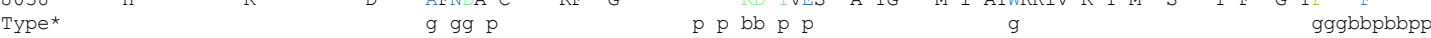

Light chain

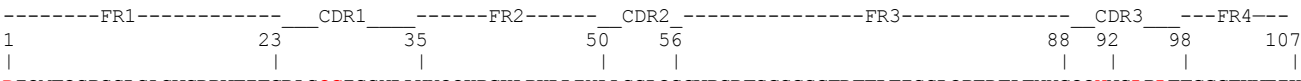

UCA DIQMTQSPSSLSASVGDRVTITCRASQGISSYLAWYQQKPGKAPKLLIYAASSLQSGVPSRFSGSGSGTDFTLTISSLQPEDFATYYCQQYNSLPLTFGGGTKVEIK

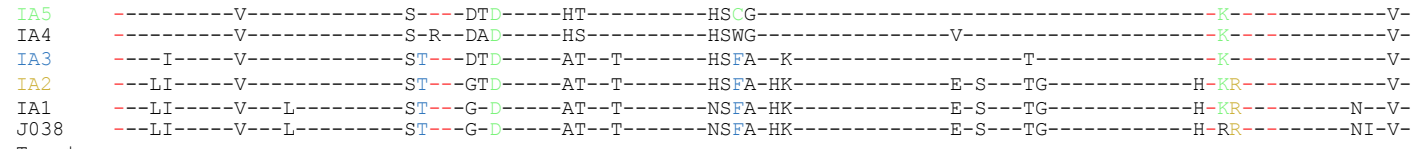

Type* $\mathrm{p} \quad \mathrm{ppp} p \mathrm{p} \quad \mathrm{g}$ pppp

* Paratope residue and type: Protein only contact: p, glycan only contact: g, protein and glycan contact: b

D Mapping of evolution of paratope residues on models of inferred intermediate antibodies
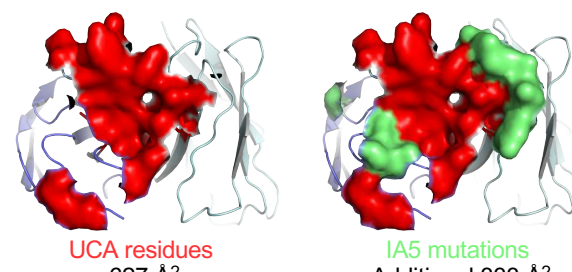

Additional $333 \AA^{2}$
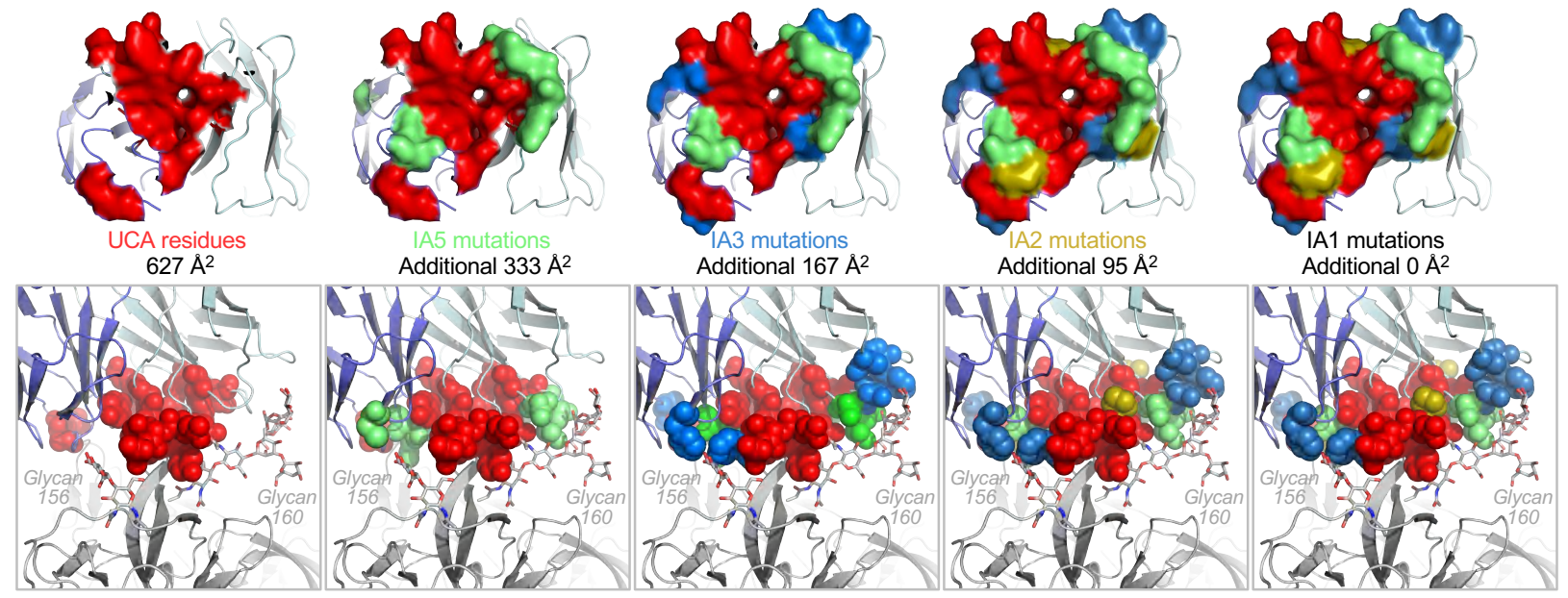

Figure 6. Development of neutralization breadth during maturation of the J033 lineage Abs.

(A) The maximum likelihood phylogenetic tree of the J038 lineage sequences. UCA and IAs at evolutionary nodes were computationally inferred. Two different clades are shaded with different colors.

(B) Neutralization profiles of UCA and IAs were determined with 17 tier 2 viruses, three tier 1 viruses and the autologous virus. Murine leukemia virus (MLV) was used as a nonspecific control. Neutralization titers are represented as $\mathrm{IC}_{50}(\mu \mathrm{g} / \mathrm{mL})$.

(C) Sequence alignment of the UCA, intermediates and mature J038 Ab sequences. Heavy chain shown in the upper set and light chain shown in the lower set. Env contacts are shown as $\mathrm{g}$ (glycan contacts), $\mathrm{s}$ (side chain contacts) and b (both side chain and main chain contacts).

(D) Mapping of the development of J038 paratope residues on models of inferred intermediate antibodies. Paratope residues directly from UCA were colored red, those from IA5, IA3 and IA2 were colored green, light blue and olive, respectively. Paratope surface areas estimated from UCA and increase from intermediate antibodies are indicated. Top panels: on antibody. Bottom panels: interface of antibody and V2-apex.

See also Figures S7 and Table S5. 
A Evolution of the V2 sequences in G1015R

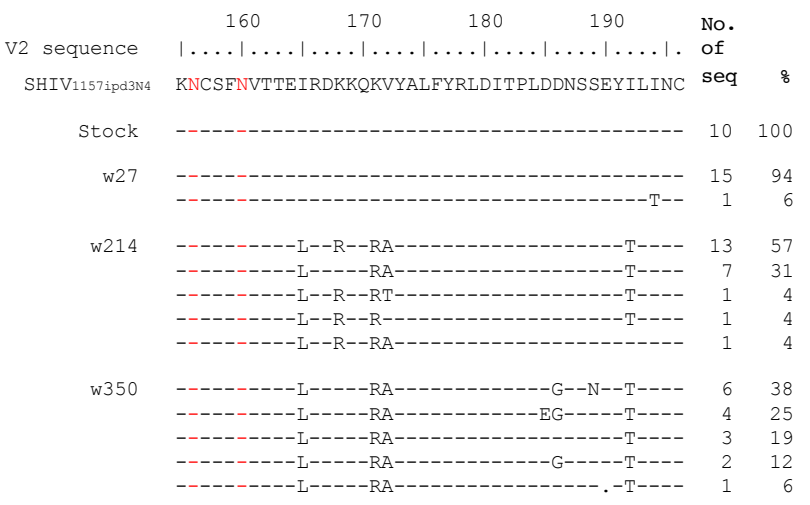

B Effects of autologous mutations on neutralization of J038 lineage antibodies

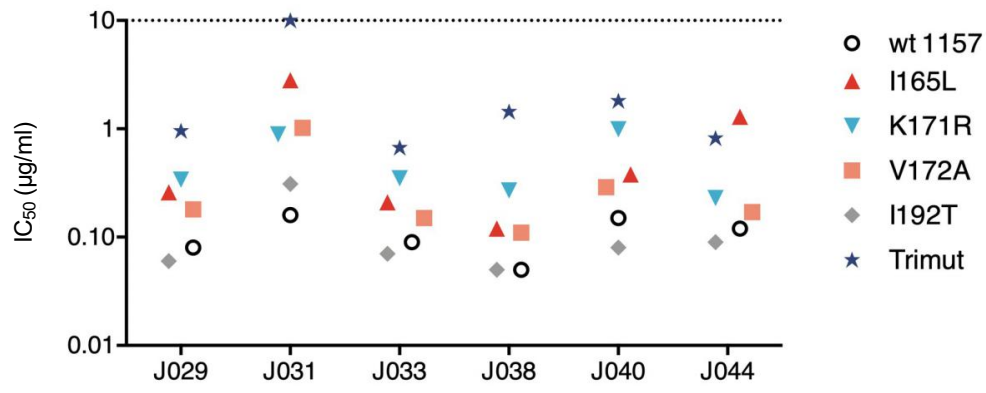

C Structure analysis of autologous escape mutations
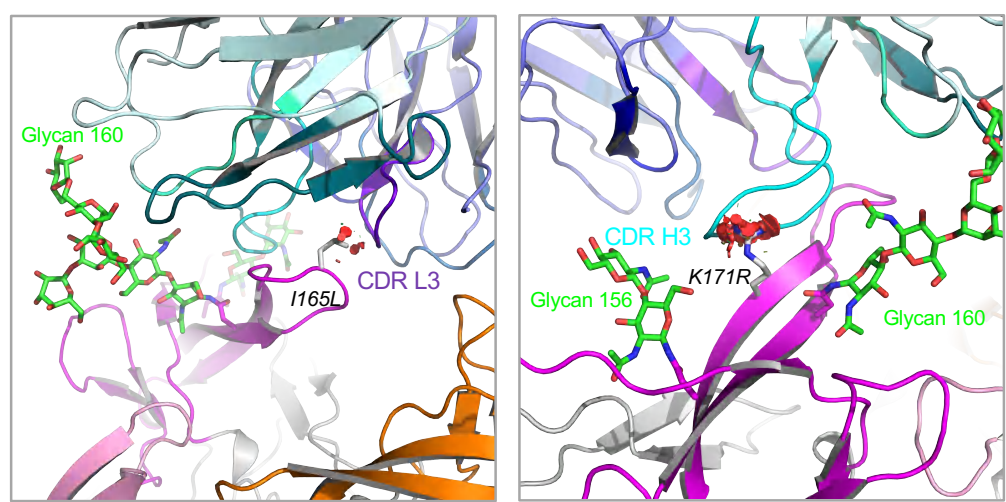

Figure 7. Limited mutations accumulated at the J038-Env contact site during infection.

(A) Identification of neutralization escape mutations in the env gene from longitudinal samples in G1015R. Amino acid sequences of V2 from the viral stock and weeks 27, 214 and 350 post infection were compared to the SHIV ${ }_{1157 i p d 3 N 4}$ reference sequence. The amino acid positions are based on the HIV-1 HXB2 sequence.

(B) Effects of the mutations in V2 on neutralization potency of the J038 lineage antibodies. 1165L, K171R and $\mathrm{V} 172 \mathrm{~A}$ render the virus resistant to J038 lineage antibodies at various levels.

(C) Structural basis for neutralization resistance by the I165L and K171R mutations. Leu165 and Arg171 are shown in stick representation. Potential clashes with CDR I3 and CDR H3 caused by these mutations are indicated as red discs. 